\title{
Der lumbale Bandscheibenvorfall
}

\author{
H. M. Mayer, F. C. Heider
}

Schön Klinik München-Harlaching, Akademisches Lehrkrankenhaus der Paracelsus Univ. Salzburg;

FIFA Medical Centre of Excellence, Wirbelsäulenzentrum

Der lumbale Bandscheibenvorfall ist eine Verlaufsvariante der Bandscheibendegeneration und stellt das häufigste Krankheitsbild der Lendenwirbelsäule im mittleren Lebensalter dar. Die klinischen Symptome des lumbalen Bandscheibenvorfalls reichen von einer reinen Schmerzsymptomatik über Sensibilitätsstörungen bis hin zu motorischen Ausfallerscheinungen, wobei als Leitsymptom eine radikuläre Symptomatik durch Druck auf einen oder mehrere Spinalnerven gilt.

Dieser Beitrag beleuchtet die diagnostischen und therapeutischen Techniken und Besonderheiten dieses sozialmedizinisch so bedeutsamen Krankheitsbilds.

\section{Einleitung}

Degenerative Veränderungen der Lendenwirbelsäule und die damit einhergehenden symptomatischen Krankheitsbilder zählen zu den am häufigsten auftretenden Beschwerden in den Industrieländern der westlichen Welt. Die Jahresprävalenz von behandlungsbedürftigen Beschwerden liegt in Deutschland zwischen 45 und 65\%. Statistiken belegen, dass nahezu jeder 2. Hausarztbesuch wegen Wirbelsäulenbeschwerden erfolgt und dass nahezu die Hälfte aller vorzeitigen Berentungen aus dem gleichen Grund beantragt wird. Hieraus wird die sozialmedizinische Bedeutung degenerativer Wirbelsäulenveränderungen ersichtlich. Die Summe aus direkten (Akutbehandlung, ambulant, stationär) und indirekten (Arbeitsunfähigkeit, vorzeitige Berentung etc.) Krankheitskosten liegt in Deutschland bei mehr als 20 Milliarden $€$ jährlich.

Der lumbale Bandscheibenvorfall ist eine Verlaufsvariante der Bandscheibendegeneration und stellt das häufigste Krankheitsbild der Lendenwirbelsäule im mittleren Lebensalter dar.

\section{Epidemiologie und Ätiologie}

Die Jahresprävalenz lumbaler Bandscheibenvorfälle liegt in den westlichen Industrieländern im Durchschnitt zwischen 1 und 2,5\%, zeigt aber eine deutliche Altersabhängigkeit mit einem Gipfel zwischen dem 30. und 50. Lebensjahr. Die Lebenszeitprävalenz beträgt bei unter 35-Jährigen 3,5\% und steigt bei 45- bis 55-Jährigen auf über $20 \%$ an. Bandscheibenvorfälle bei Kindern sind selten, verlässliche Prävalenzdaten liegen nicht vor. Unter den operativ behandelten Fällen sind Kinder und Jugendliche nur mit 0,2-3,2\% vertreten.

\section{Abkürzungen}

$\begin{array}{ll}\text { AU } & \text { Arbeitsunfähigkeit } \\ \text { BSV } & \text { Bandscheibenvorfall } \\ \text { BU } & \text { Berufsunfähigkeit } \\ \text { BV } & \text { Bildwandler } \\ \text { ISG } & \text { \|liosakralgelenk } \\ \text { LWS } & \text { Lendenwirbelsäule } \\ \text { MdE } & \text { Minderung der Erwerbsfähigkeit } \\ \text { WS } & \text { Wirbelsäule }\end{array}$


Lumbale Bandscheibenvorfälle zeigen eine leichte, gelegentlich ein Verhältnis von 2:1 erreichende, Geschlechtspräferenz für Männer.

Etwa $90 \%$ aller lumbalen Bandscheibenvorfälle betreffen die beiden kaudalen Bewegungssegmente (L4-L5 und L5-S1), wobei mit zunehmendem Alter häufiger die Level oberhalb von L5-S1 betroffen sind.
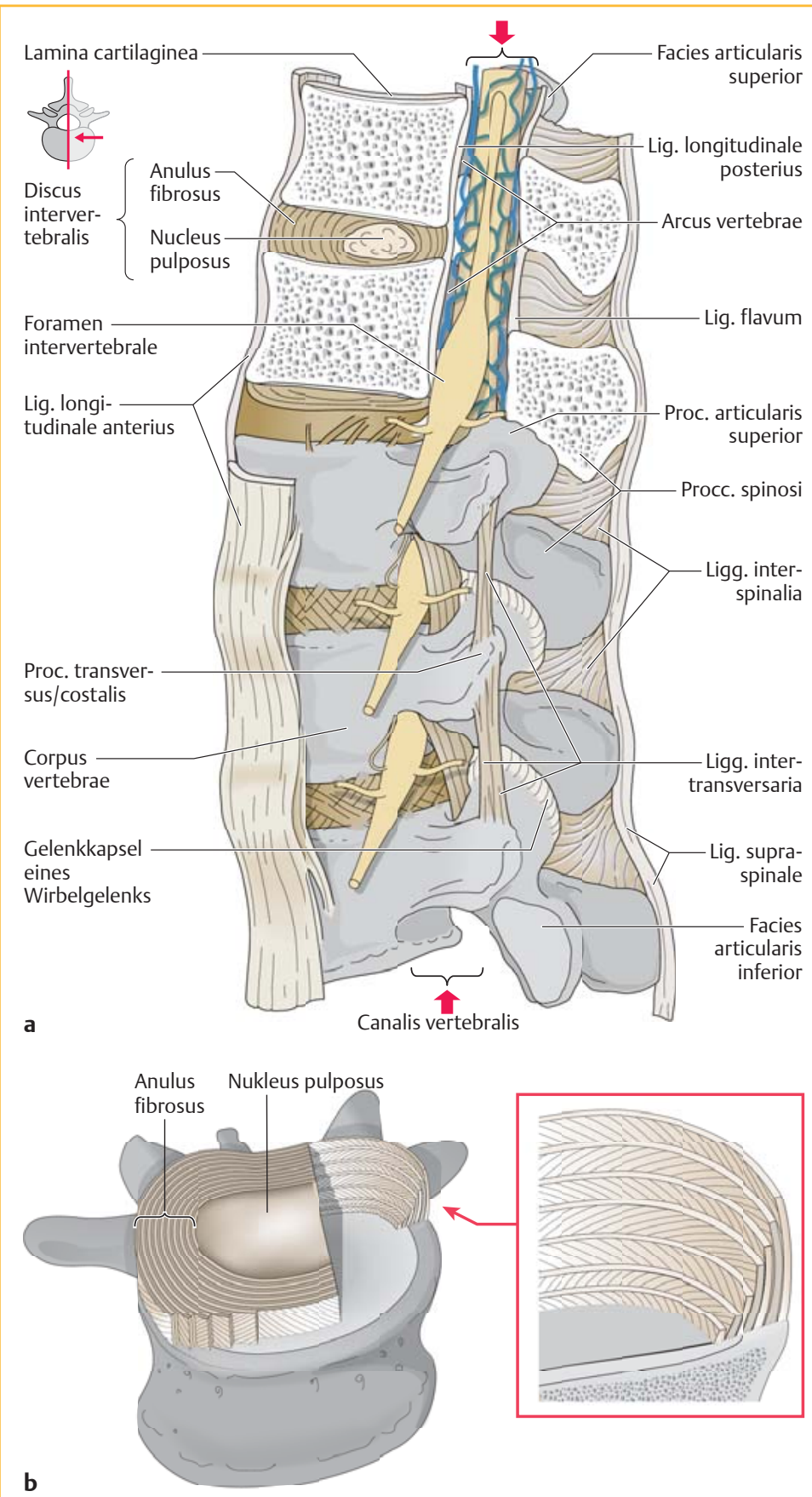

Abb. 1 - Lumbale Bandscheibe. a Anatomischer Aufbau der lumbalen Bewegungssegmente mit Darstellung der Lagebeziehung zu den abgehenden Spinalnervenwurzeln. b Schematische Darstellung des anatomischen Aufbaus einer gesunden Bandscheibe.

\section{Pathophysiologie der Bandscheibendegeneration}

Die lumbale Bandscheibe ist das größte bindegewebige Organ, das ab dem Erreichen des Zweifüßlergangs über keine eigene Blutversorgung mehr verfügt (Abb. 1).

Diese Tatsache und das Zusammenwirken weiterer Faktoren wie Alter, Gewicht, (sportliche oder berufliche) biomechanische Belastung sowie anatomische (Lendenlordose) und konstitutionelle Faktoren (genetische Prädisposition) beeinflussen den bereits im frühen Kindesalter einsetzenden Degenerationsprozess der Bandscheibe. An dessen Beginn stehen Rissbildungen im Anulus fibrosus (Abb. 2), begünstigt durch

- Umstrukturierung kollagener Fasern,

- mechanische Belastungen,

- Abnahme der Proteoglykankonzentration mit

- einhergehenden qualitativen Proteoglykanveränderungen und

- einer konsekutiven Abnahme des Flüssigkeitsgehalts des Nucleus pulposus (Abb. 2).

Aufgrund der fehlenden Blutversorgung heilen Anulusrisse nicht aus. Die Bandscheibe „trocknet aus“, verliert an Höhe und kann ihrer biomechanischen Funktion nicht mehr gerecht werden. Dies führt zu einer alterierten Verteilung von axialen, rotatorischen und translatorischen Kräften, was den weiteren Degenerationsvorgang begünstigt. Translatorische und vertikale Instabilitäten, die aus einem Höhenverlust der Bandscheibe und damit einem Verlust der ligamentären Grundspannung resultieren, verstärken die biomechanische Fehlbelastung und damit den degenerativen Selbstzerstörungsprozess der Bandscheibe.

Dieser stadienhafte Ablauf einer Bandscheibendegeneration hat zu unterschiedlichen Klassifizierungen geführt, wobei die kernspintomografische Klassifizierung nach Pfirrmann (Abb. 3) die größte internationale Akzeptanz besitzt.

In dieser degenerativen Kaskade gibt es pathomorphologische und biomechanische Konstellationen, die die Entstehung eines Bandscheibenvorfalls begünstigen.

Vor allem das Zusammentreffen von noch ausreichend hydriertem Nucleus pulposus mit bereits fortschreitender Degeneration im Anulus fibrosus (z. B. Pfirrmann-Stadien II, III und IV) begünstigen die Entstehung eines Bandscheibenvorfalls. 


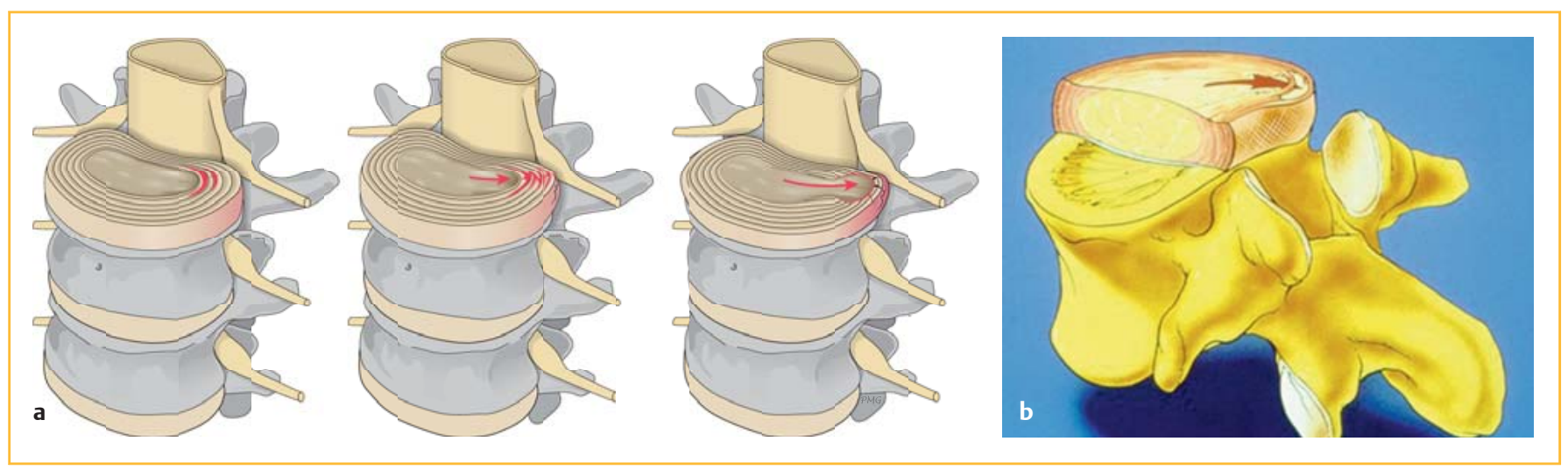

Abb. 2 - Lumbale Bandscheibenrissbildung im Anulus fibrosus. a Schematische Darstellung der Bandscheibendegeneration mit Ausbildung einer Rissbildung im Anulus fibrosus bis hin zur beginnenden Protrusion des Nukleus pulposus (von links nach rechts). b Schematische Darstellung einer Anulusläsion (Pfeil).
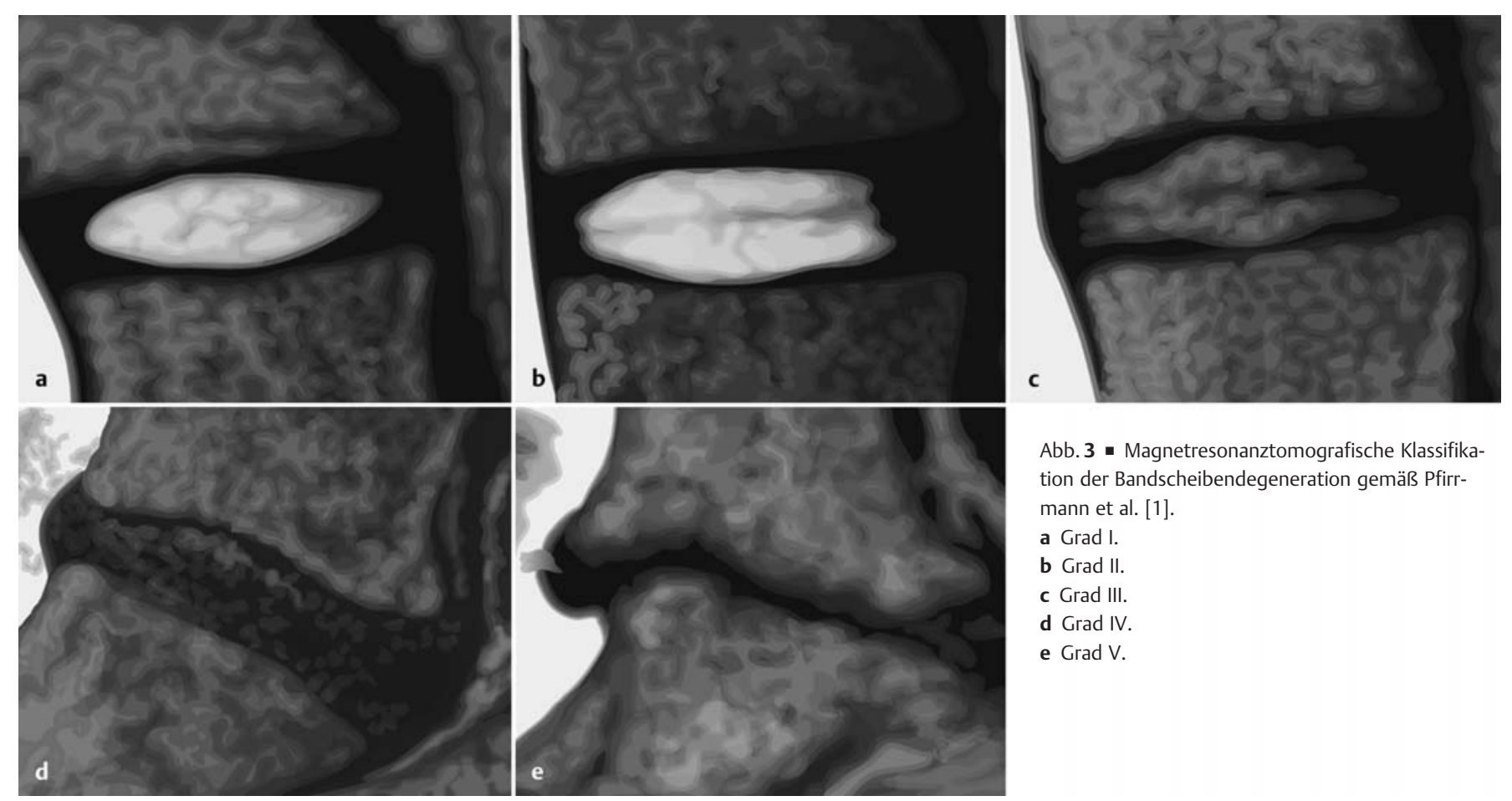

Abb. 3 - Magnetresonanztomografische Klassifikation der Bandscheibendegeneration gemäß Pfirrmann et al. [1].

a Grad I.

b Grad II.

c Grad III.

d Grad IV.

e Grad V.

Da diese Stadien II, III und IVi.d. R. im mittleren Lebensabschnitt auftreten, erklärt dies auch die Häufung von Bandscheibenvorfällen (BSV) zwischen dem 30. und 50. Lebensjahr.

\section{Pathogenese des lumbalen Bandscheibenvorfalls}

- Die Entstehung eines Bandscheibenvorfalls setzt i. d. R. einen begünstigenden Degenerationszustand der Bandscheibe voraus.
Traumatisch bedingte Bandscheibenvorfälle in ansonsten gesunden Bandscheiben sind zwar möglich, aber sehr selten. Sie setzen akute oder wiederholte Extrembelastungen im Sinne von Hyperflexion/Kompression oder Flexion/Rotation voraus, wie sie nur bei Unfällen oder bei repetitiver Überlastung im Hochleistungssport (z.B. Turnen, Fußball, Baseball etc.; Abb. 4) vorkommen.

Hierbei kommt es entweder zu einer traumatischen Rissbildung oder Desintegration der Anulus-fibrosusLamellen mit direkter Herniation von weichen Nucleuspulposus-Anteilen (Abb. 4) oder aber nur zu einer Riss- 


\section{Wirbelsäule}

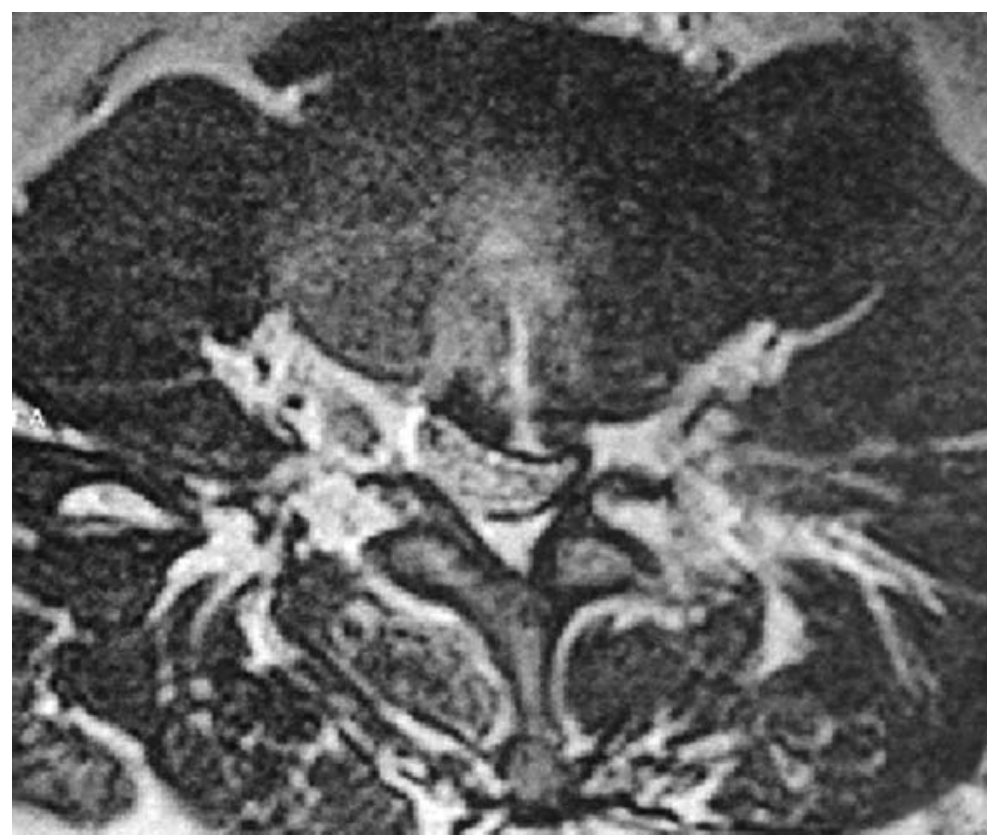

Abb. 4 - MRT: Riss im Anulus und BSV bei einem Fußballer. bildung, die dann durch fehlendes Heilungspotenzial und weitere Belastung zu einer Schwachstelle wird, durch die Nucleus-pulposus-Gewebe austreten kann.

Einen Bandscheibenvorfall auslösende Bewegungsmuster sind unter biomechanischen Gesichtspunkten in erster Linie folgende:

- forcierte Hyperflexion kombiniert mit Kompression und/oder

- Rotation oder

- zyklische Flexion/Kompression/Rotation.

Durch die Kompression der vorderen Bandscheibenanteile kommt es zu einer akuten oder langsamen Volumenverlagerung des Nucleus pulposus nach dorsal in Richtung des geschwächten Anulus. Darüber hinaus wird durch diese Bewegungsmuster die Distorsion der Anuluslamellen und damit die Rissbildung begünstigt. Im Alltag sind dies Bewegungen wie z.B. das Anheben eines

Fazit

\section{Prinzipien}

Da sich der Nukleus unter axialer Belastung zu einer Verteilung der einwirkenden Last in alle Richtungen ausdehnt, folgt er stets dem Weg des geringsten Widerstands (in diesem
Fall dem defekten Anulus fibrosus). Dies kann sowohl zu einer Anulusvorwölbung (Protrusion) (Abb. 5) als auch zu einem Austritt von Nukleusgewebe durch den Anulus in den sub-
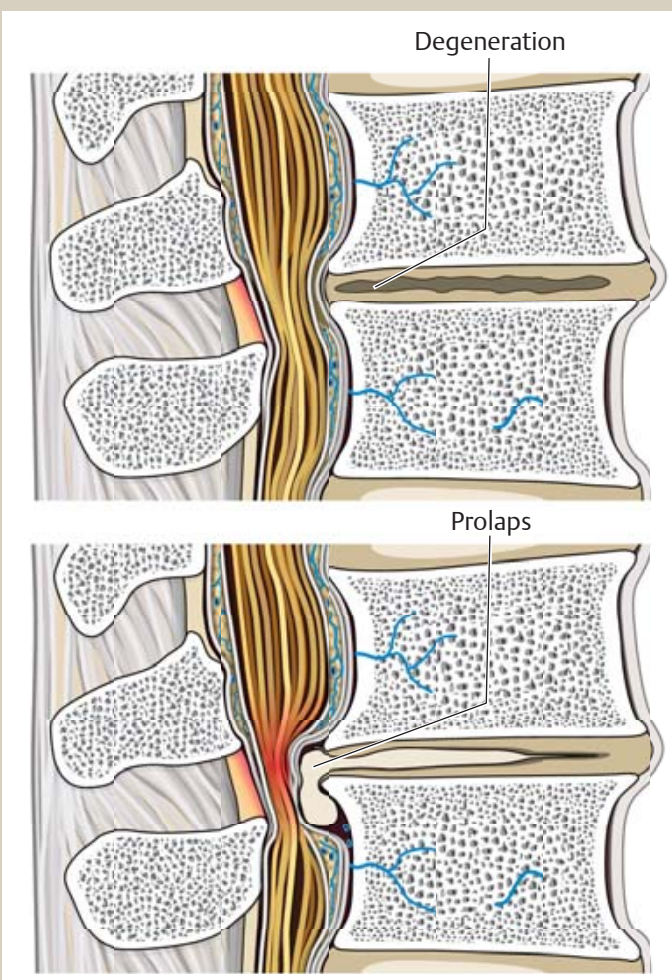
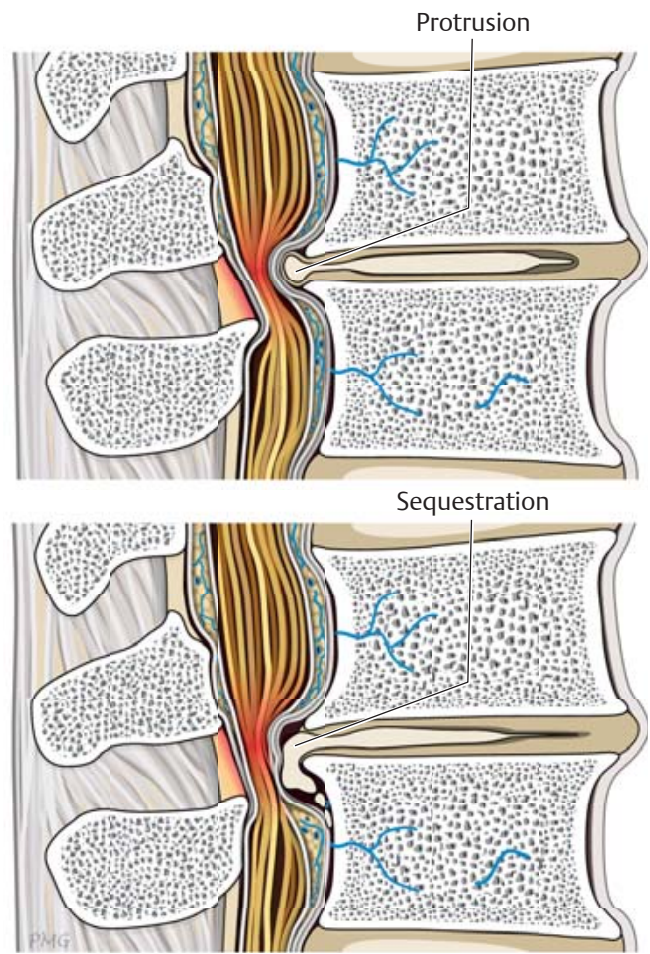

Abb. 5 - Schema des Bandscheibenvorfalls: Protrusion - Sequester. 
Gegenstands mit Rotation des Oberkörpers oder das weite Vorbeugen, um einen Gegenstand vom Boden aufzuheben (Abb. 6).

\section{Anamnese und Symptomatik}

Für die klinische Symptomatik des Bandscheibenvorfalls sind mehrere Faktoren verantwortlich. Zunächst kommt es durch die Vorwölbung der Bandscheibe zu einer mechanischen Irritation oder Kompression der benachbarten Nervenstrukturen. In Tierexperimenten und klinischen Versuchen konnte gezeigt werden, dass die Berührung bzw. der Druck auf einen gesunden Spinalnerv i.d.R. mit Parästhesien oder motorischen Störungen einhergeht, aber nicht primär schmerzhaft sein muss.

Die Schmerzentstehung ist eine Kombination von mechanischer Irritation und der dadurch ausgelösten lokalen Ausschüttung von Entzündungsmediatoren. Diese Ausschüttung erfolgt verstärkt, wenn Nucleuspulposus-Material in den Epiduralraum austritt.

In der Regel berichtet die überwiegende Mehrzahl der Patienten anamnestisch über intermittierende oder chronische Lumbalgien, die dem Akutereignis Bandscheibenvorfall vorausgehen. Die Entstehung dieser Lumbalgien ist multifaktoriell. Da die Bandscheibe und deren Umgebungsstrukturen eine komplexe Innervation aufweisen, kommen mehrere Strukturen als Auslöser der Rückenschmerzen infrage (Abb. 7).

Degenerative Veränderungen des Anulus fibrosus wie auch der knorpeligen Grund- und Deckplatten induzieren das Einsprossen von sensiblen Nerven und die Mikrovaskularisation. Dies kann unter Druckbelastung lokale Rückenschmerzen verursachen. Gleiches gilt für die Dehnung der äußeren Anulusfasern in der Phase der Bandscheibenprotrusion oder die Irritation der Dura mater ohne Nervenkompression (Abb. 8).

Die Degeneration der Bandscheibe geht mit einer Höhenminderung und demzufolge mit einer erhöhten Belastung der Wirbelgelenke einher. Dies induziert Knorpelschäden an den Gelenkflächen bzw. eine Spondylarthrose, die ebenfalls zu Lumbalgien führen kann.

Das Leitsymptom des Bandscheibenvorfalls ist der allgemein als Ischialgie bezeichnete, ins Bein ausstrahlende Schmerz, der mit oder ohne begleitende neurologische Ausfälle auftreten kann.

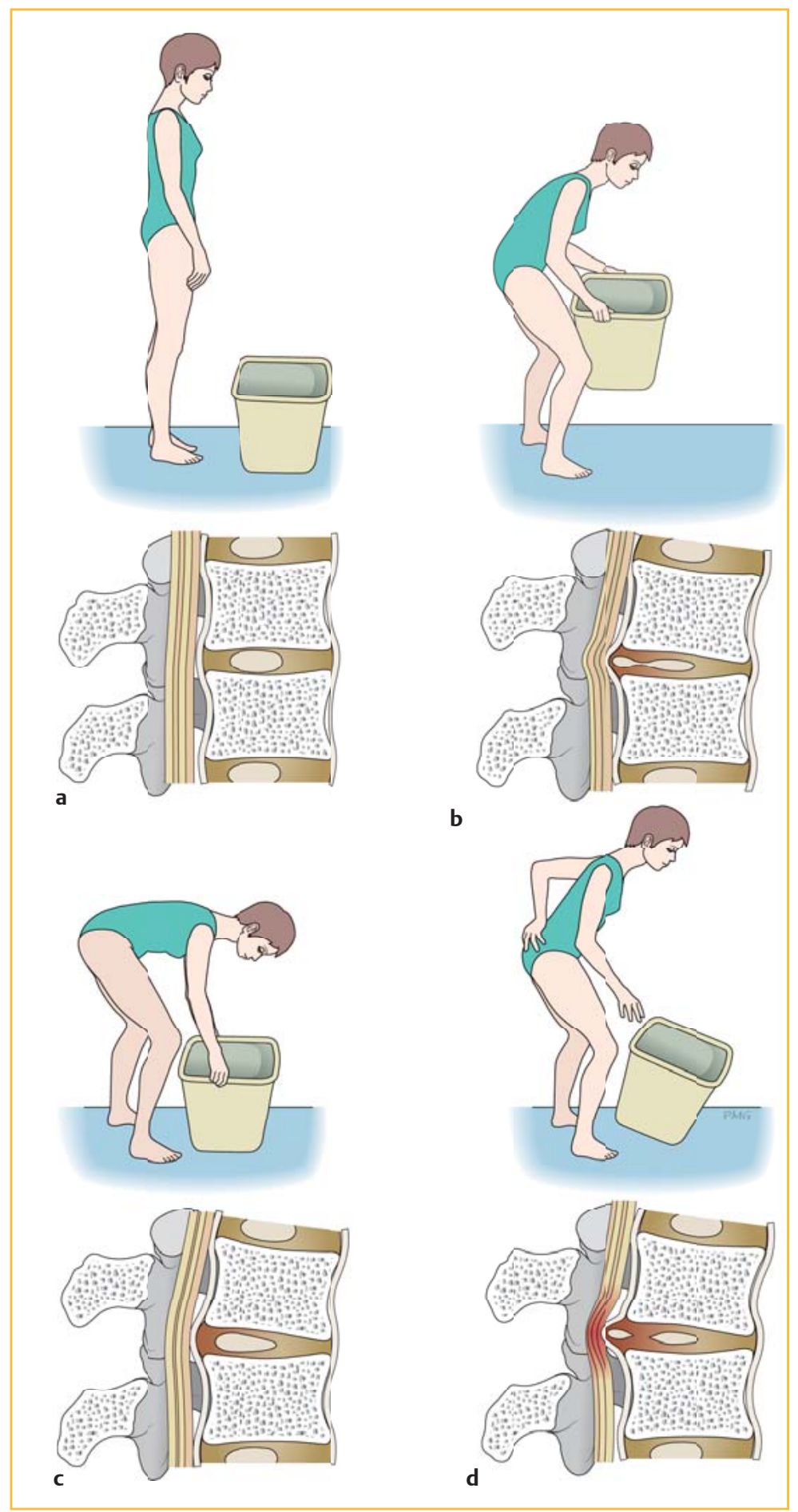

Abb. 6 - Typisches Bewegungsmuster, das durch eine vermehrte anteriore Kompression des Bandscheibenbewegungssegments einen BSV auslösen kann. a Ausgangssituation; b,c Hyperflexion mit Rotation; d ausgelöster Schmerzzustand bei manifestem BSV. 


\section{Wirbelsäule}

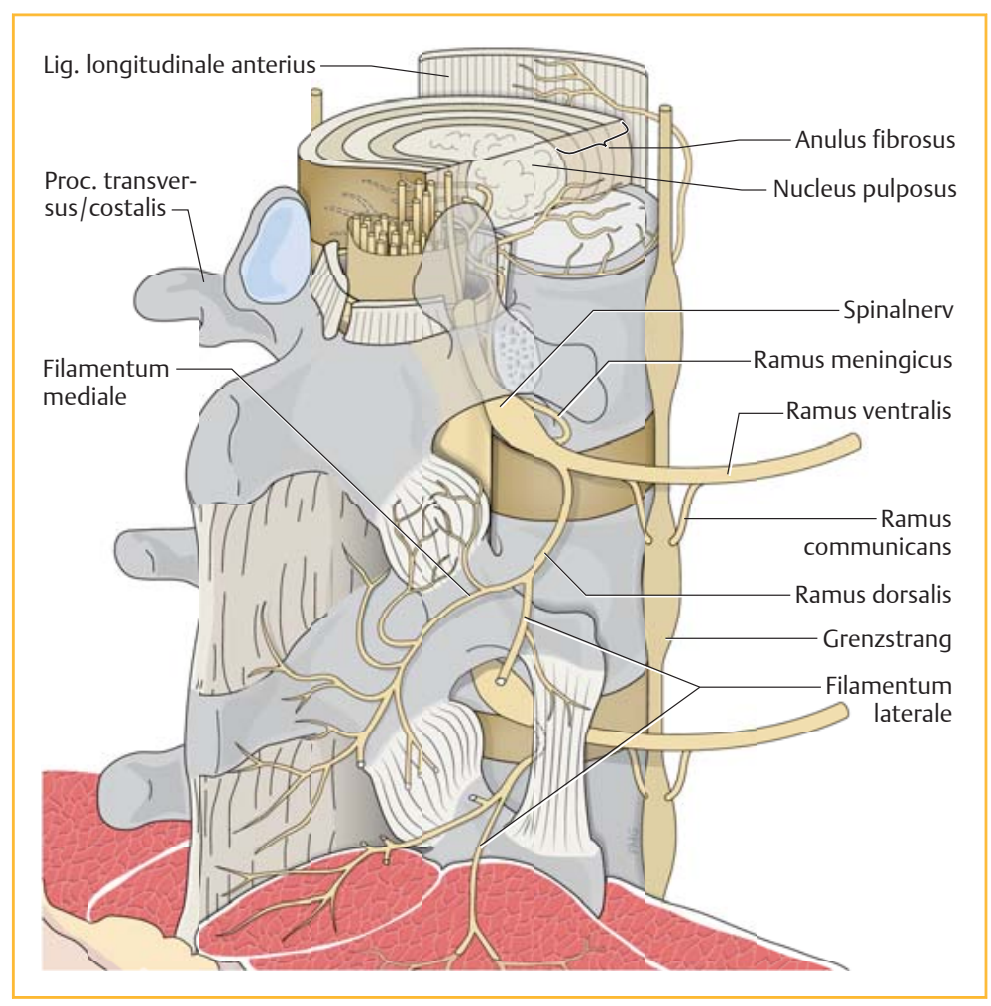

Abb. 7 - Innervation Bewegungssegment.

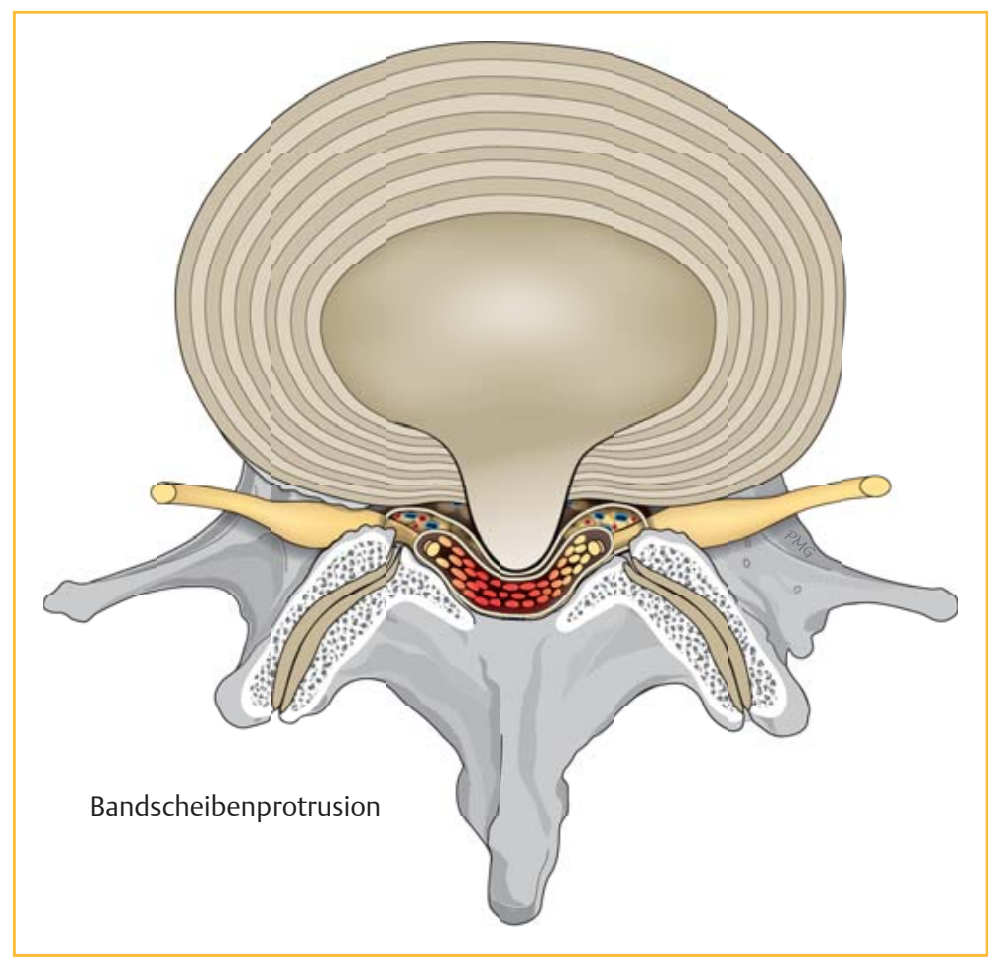

Abb. 8 - Große mediale Protrusion mit Durairritation.
Abhängig von der Lokalisation, Ausdehnungsrichtung und Größe des Bandscheibenvorfalls können folgende Strukturen betroffen sein:

- einzelne oder mehrere Spinalnerven,

- das Spinalganglion,

- die Cauda equina,

- der Conus medullaris.

Durch die Anamneseerhebung erhält man wichtige Hinweise zur Differenzialdiagnostik bzw. zur wahrscheinlichen Pathomorphologie und Lokalisation des Bandscheibenvorfalls (s. Infobox „Zielgerichtete Diagnostik“).

\section{Zielgerichtete Diagnostik}

\section{Anamnese}

\section{Folgende Fragen sind wichtig}

- Familienanamnese

- Rückenschmerz

- Bandscheibenvorfall

- Beginn der Symptome

- akut/schleichend

- auslösendes Ereignis

- Lokalisation der Schmerzen

- rechts/links

- Rücken, Bein

- Dermatom

- Art der Schmerzen

- Schmerzverstärkung

- lageabhängig,

- bewegungsabhängig

- Husten, Niesen, Pressen

- Entlastungspositionen

- Begleitschmerzen (Rücken, Gesäß, Muskulatur)

- neurologische Symptome

- Sensibilitätsstörungen

- Schwächegefühl (wo, wobei)

- Probleme beim Wasserlassen/Stuhlgang (Harnverhalt, Urgeinkontinenz, Stuhlinkontinenz)

- Einfluss der Symptome auf die individuelle Lebensqualität

- sozioökonomische Aspekte

- Beruf

- Familienstand

- Sport

- Berentung, Arbeitsfähigkeit, Dauer der AU/BU, laufendes Rentenbegehren, MdE, Schwerbehinderung etc.)

- bisherige Therapiemaßnahmen 


\section{Der lumbale Bandscheibenvorfall}

\section{Diagnostisches Vorgehen}

\section{Klinische Untersuchung}

Die Schritte der klinischen Untersuchung sind in einer Infobox zusammengefasst (s.a. Tab. 1 u. Abb. 9-12).

\section{Zielgerichtete Diagnostik}

\section{Klinische Untersuchung}

Klinische Inspektion

- Haltung der WS

- Schonhaltung (Abb. 9)

- Tilt

- Kyphose

- Skoliose

- Spondylolisthese

- Becken- und Beinposition

\section{Bewegungseinschränkung}

- muskulärer Hartspann, Myogelosen

- Schmerzprojektion

- Dermatom(e),

- „pseudoradikuläre“ Schmerzen

Neurologische Untersuchung der unteren Extremität

- (Tab. 1)

- klinische Zeichen:

- Lasègue-Test $\rightarrow$ radikuläre Symptome bei Anheben des gestreckten Beines (Abb. 10)

- Mennell-Test $\rightarrow$ zum Ausschluss einer Iliosakralgelenkirritation (Abb. 11)

- Viererzeichen $\rightarrow$ Facettenschmerz, ausgelöst durch Rotation bei Abduktion der gebeugten Hüfte mit fixiertem Becken (Abb. 12)

\section{Tabelle 1}

Segmentale neurologisch-motorische Zugehörigkeit bei Schädigung der lumbalen Nervenwurzeln (die Muskelgruppen sind nach Wurzel und Funktion zusammengefasst).

\begin{tabular}{|ll|}
\hline Sement & Motorik \\
\hline L2/L3 & Hüftbeuger (Oberschenkel heben im Sitzen) \\
\hline L4 & Kniestrecker (Kniebeuge aus dem Stand) \\
\hline L5 & Fuß- und Zehenhebemuskeln (Hackengang) \\
\hline S1 & Plantarflektoren (Zehengang) \\
\hline
\end{tabular}

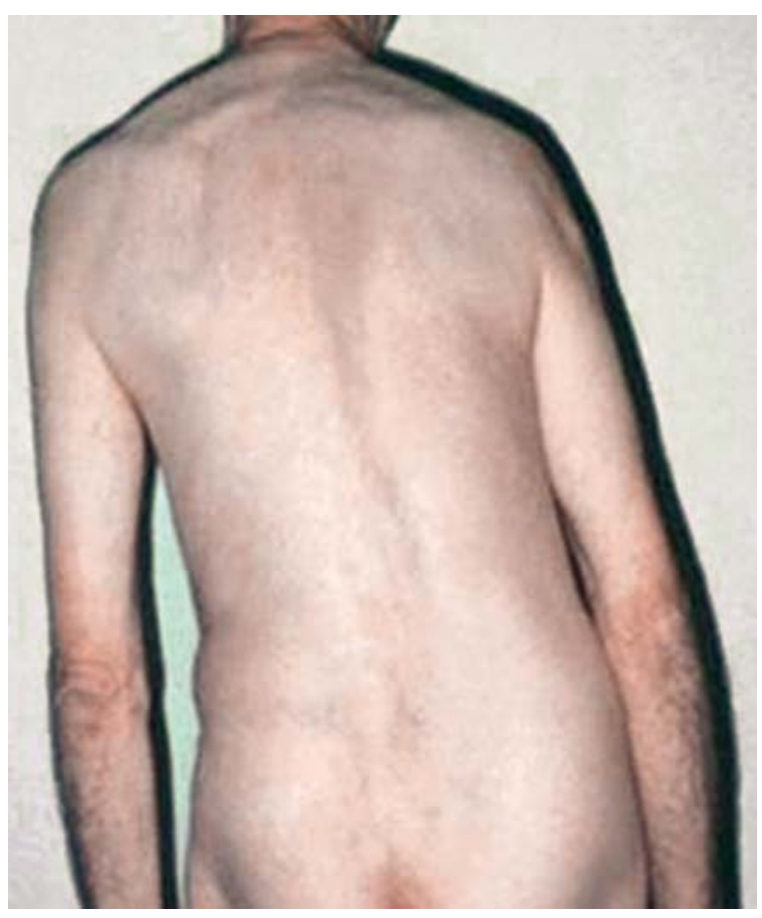

Abb. 9 - Typische Schonhaltung bei lumbalem Bandscheibenvorfall.

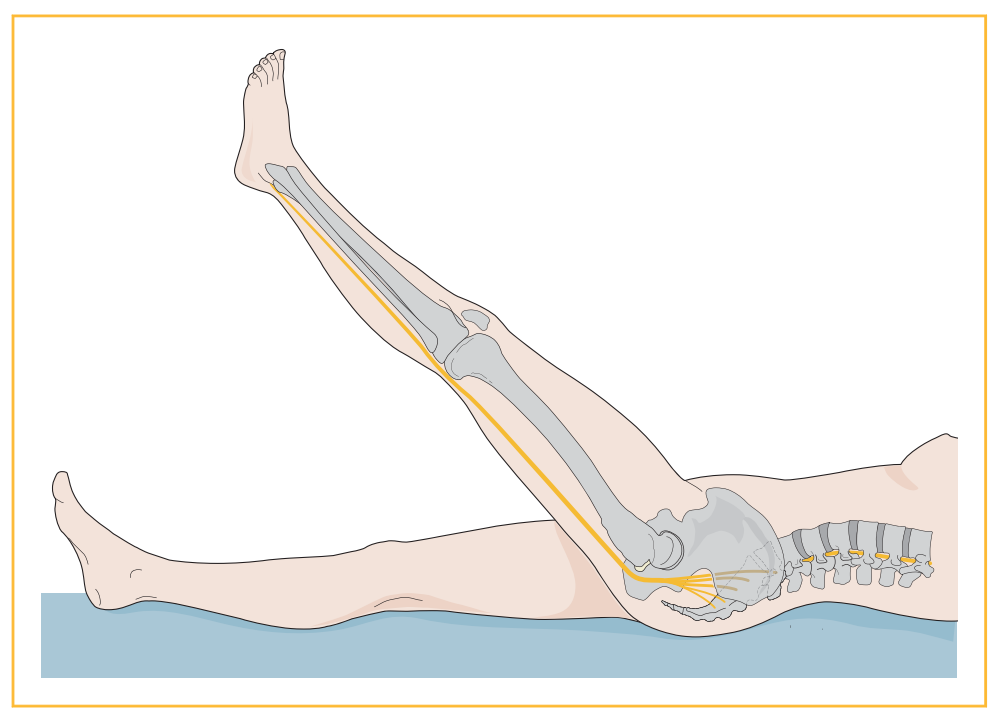

Abb. 10 - Lasègue-Test: Auslösung radikulärer Symptome bei Anheben des gestreckten Beines. 


\section{Wirbelsäule}

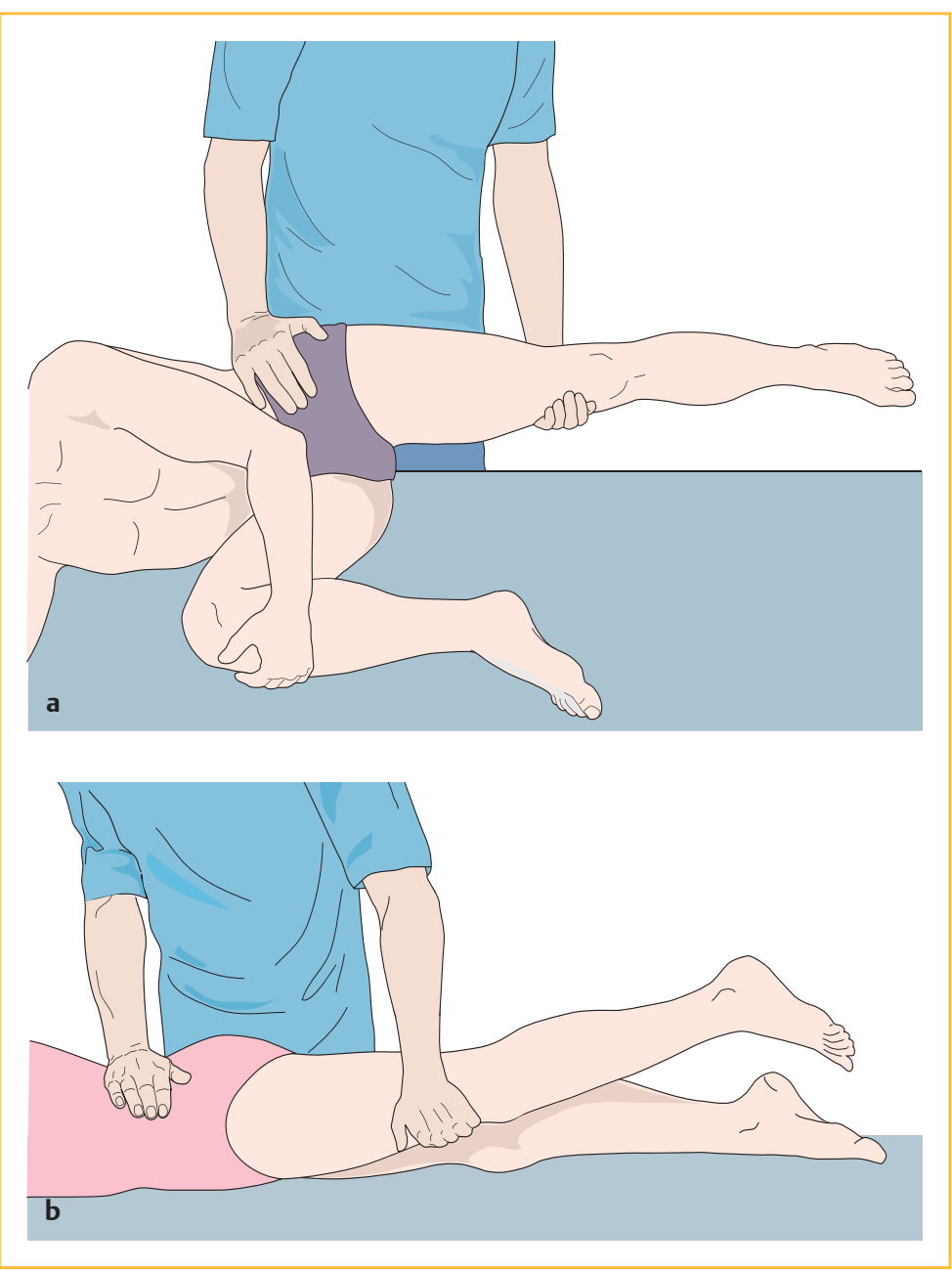

Abb. 11 - Der Mennell-Test dient zum Ausschluss einer ISG. a Testung in Seitenlage. b Testung in Bauchlage.

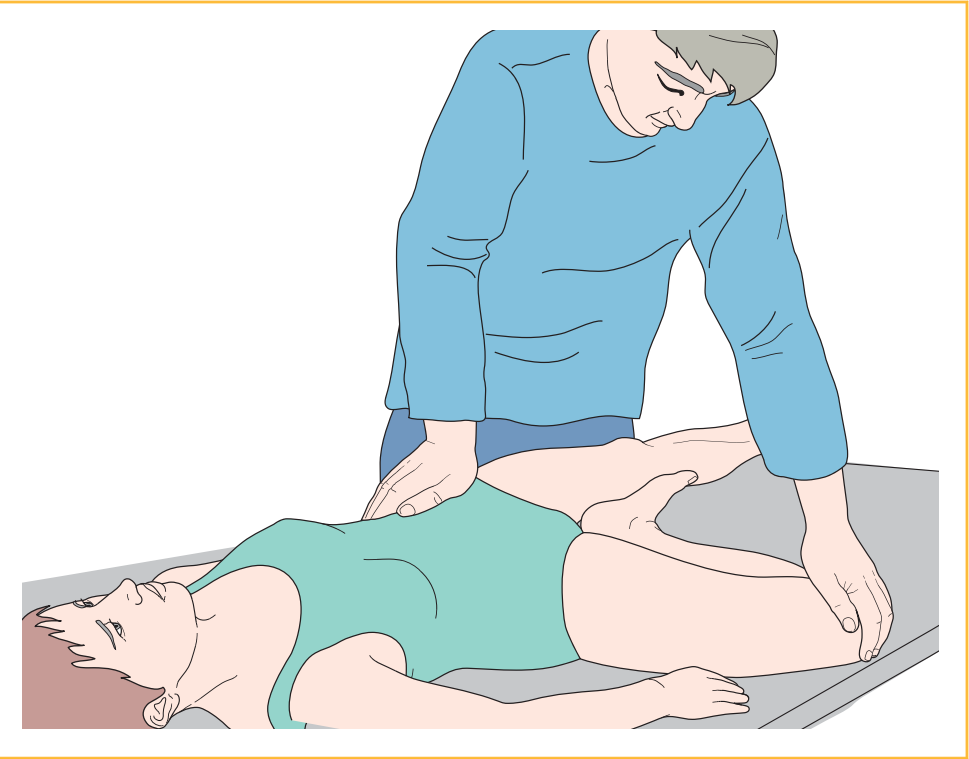

Abb. 12 - Beim Viererzeichen imponiert ein Facettenschmerz, ausgelöst durch Rotation bei Abduktion der gebeugten Hüfte mit fixiertem Becken.

\section{Diagnosesicherung}

MRT. Die Diagnosesicherung erfolgt mittels Kernspintomografie (MRT; Abb. 13). Das detaillierte Vorgehen ist in einer weiteren Infobox „Zielgerichtete Diagnostik“ zusammengefasst.

\begin{tabular}{l} 
Zielgerichtete Diagnostik \\
\hline Diagnosesicherung \\
\hline Kernspintomografie \\
- Lokalisation und Ausdehnung des Bandscheibenvorfalls \\
(Abb. 13) \\
- Etage \\
- medial, paramedian, rezessal, foraminal, extraforaminal \\
- Ausdehnung nach kaudal - kranial \\
Morphologie \\
- Protrusion, Prolaps, Sequester? \\
- frisch/alt \\
- weich/hart \\
- Deckplattenmaterial? \\
Ausmaß der Nervenkompression \\
- Spinalnerv(en) \\
- Cauda equina \\
- Conus medullaris \\
Sonstige Untersuchungen \\
- Begleitpathologien \\
- Stadium der Bandscheibendegeneration \\
(Pfirrmann-Klassifikation [1]) \\
Weishaupt [2]) \\
- Stadium der Spondylarthrose (Einteilung nach \\
-
\end{tabular}

Röntgen. Bei Verdacht auf Deformität (z.B. degenerative Lumbalskoliose) oder Instabilität (z.B. degenerative Spondylolisthese) ist als nächster Schritt ein Röntgenbild der LWS im Stehen in 2 Ebenen indiziert (Abb. 14).

Sonstige Untersuchungen. Eine Computertomografie bzw. eine Myelografie sind für die Primärdiagnostik des lumbalen Bandscheibenvorfalls nicht erforderlich und aufgrund der Strahlenbelastung obsolet. Lediglich bei Unmöglichkeit der MRT-Diagnostik (z. B. Herzschrittmacherpatienten) oder ergänzend bei V.a. Ossifikationen bzw. Kalzifikationen sowie bei Rezidivvorfällen (s.u.) können sie im Einzelfall wertvolle Zusatzinformationen bringen. Die Myelografie ist zum Nachweis von lateralen/ extraforaminalen Bandscheibenvorfällen zudem nicht geeignet. 

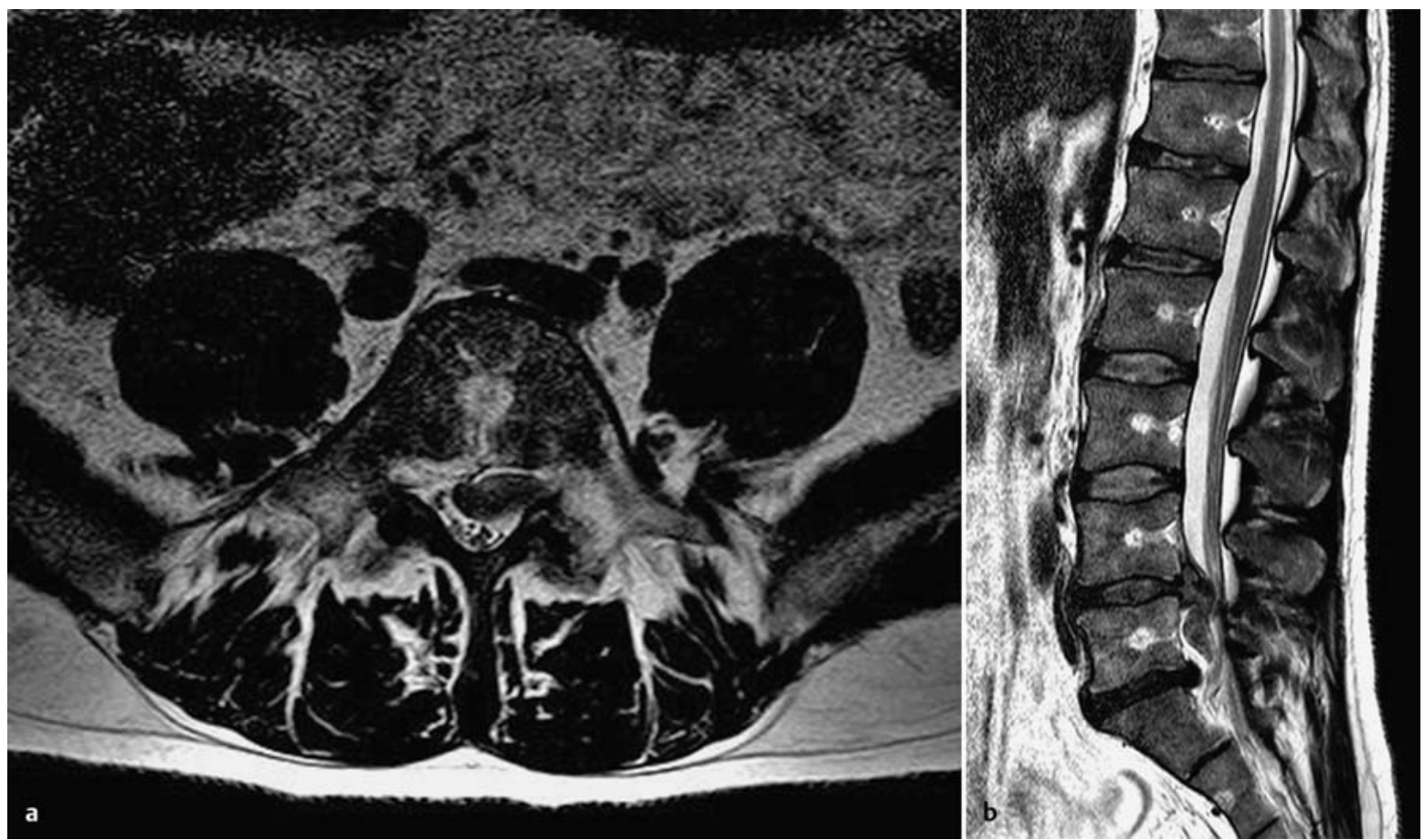

Abb. 13 - Bandscheibenvorfall im MRT. a Axial in T2-Wichtung: sequestrierter Bandscheibenprolaps L4/L5 mit Kompression des Spinalnervs L5 links. b Sagittale Schnittebene in T2-Wichtung: großer, weit nach kaudal reichender Bandscheibenprolaps L4-L5.

\section{Vorbemerkungen zum therapeutischen Vorgehen}

Bei mehr als $80 \%$ der Patienten mit einem lumbalen Bandscheibenvorfall als Erstereignis bessert sich die initiale klinische Symptomatik durch konservative Maßnahmen. Innerhalb eines Jahres erleiden ca. 30-50\% einen Rückfall, wovon dann wiederum bei etwa $50 \%$ aufgrund fehlender Besserung der Symptomatik eine OP-Indikation gestellt wird.

- Die therapeutische Strategie richtet sich nahezu ausschließlich nach der klinischen Symptomatik und sollte einem standardisierten Algorithmus folgen.

Das Therapieergebnis wird durch mehrere Faktoren bestimmt. Am wichtigsten ist das Timing der unterschiedlichen Verfahren, da die Rückbildung der Nervenkompressionssymptome zeitabhängig ist. Das bedeutet, dass die Wahl des Therapieverfahrens vom bisherigen Symptomverlauf mitbestimmt wird.

Auch zeigt nicht jeder akute Bandscheibenvorfall die gleiche Rückbildungstendenz. So zeigen neuere wissenschaftliche Untersuchungen, dass Bandscheibenprotrusionen (Anulus fibrosus intakt) eine deutlich geringere Rückbildungstendenz aufweisen als in den Epiduralraum sequestrierte Bandscheibenvorfälle (Abb. 15). Letztere werden i.d.R. besser resorbiert (Abb. 16).

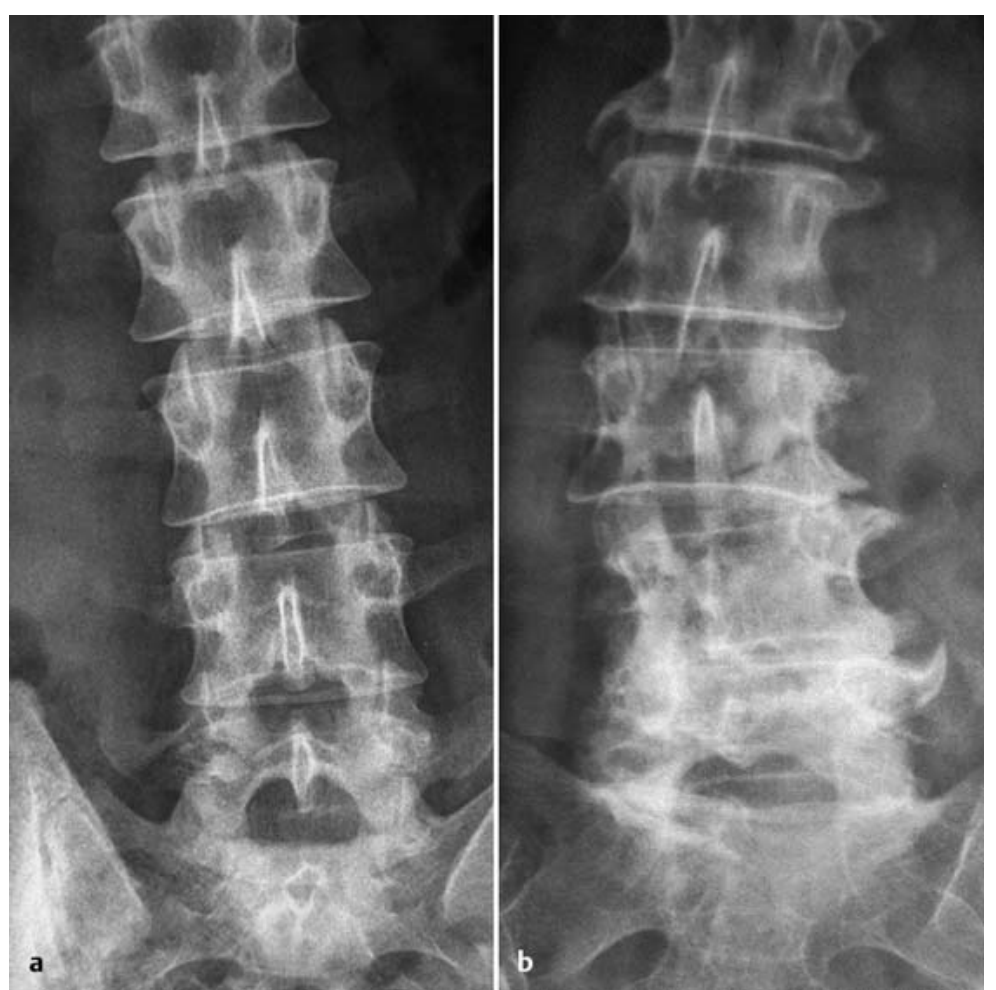

Abb. 14 - Röntgenbilder der LWS im Stehen. a Typische funktionelle linkskonvexe Fehlhaltung. b Strukturelle Deformität, degenerative Lumbalskoliose.

Auch dies sollte bei der Wahl bzw. dem Timing der Therapieverfahren berücksichtigt werden. 


\section{Wirbelsäule}

Therapie

\section{Notfalloperation}

Etwa 1-1,5\% der Patienten zeigen eine akute Konus-/Kaudasymptomatik und stellen einen absoluten operativen Notfall dar. Obgleich die wissenschaftliche Literatur bezüglich der OP-Dringlichkeit innerhalb von 24 Stunden kontrovers ist, so muss unter klinisch-praktischen Aspekten eine schnellstmögliche operative Entlastung durch Sequestrektomie propagiert werden, da die Rückbildungsfähigkeit neurologischer Ausfälle und hier insbesondere der Blasen- und Mastdarmstörungen eng mit der präoperativen Symptomdauer korreliert.

Akute Indikation im Sinne einer sofortigen OP; dringliche Indikation im Sinne von $24 \mathrm{~h}$.

\section{Therapie}

\section{Therapieziel}

Das Therapieziel ist die Rückbildung der radikulären Symptome und die soziale und berufliche Wiedereingliederung des Patienten in einem Zeitfenster von < 3 Monaten, um eine Chronifizierung der Beschwerden zu vermeiden.

Sämtliche Therapieformen sollten sich diesem Ziel unterordnen.

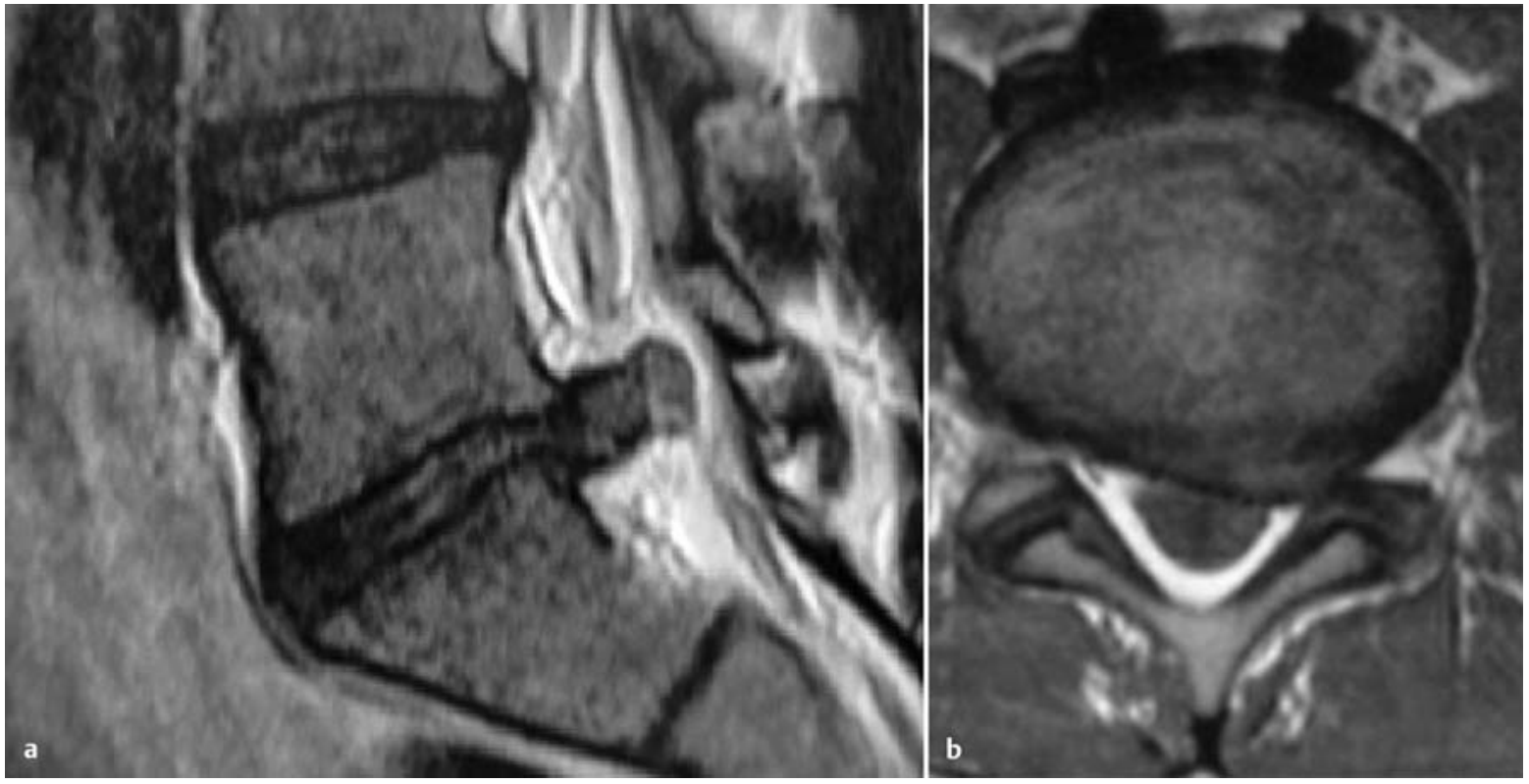

Abb. 15 - Unterschiedliche Bandscheibenvorfälle. a Sequestrierter Bandscheibenvorfall mit guter Rückbildungstendenz. b Breitbasige Protrusion mit schlechter Rückbildungstendenz.
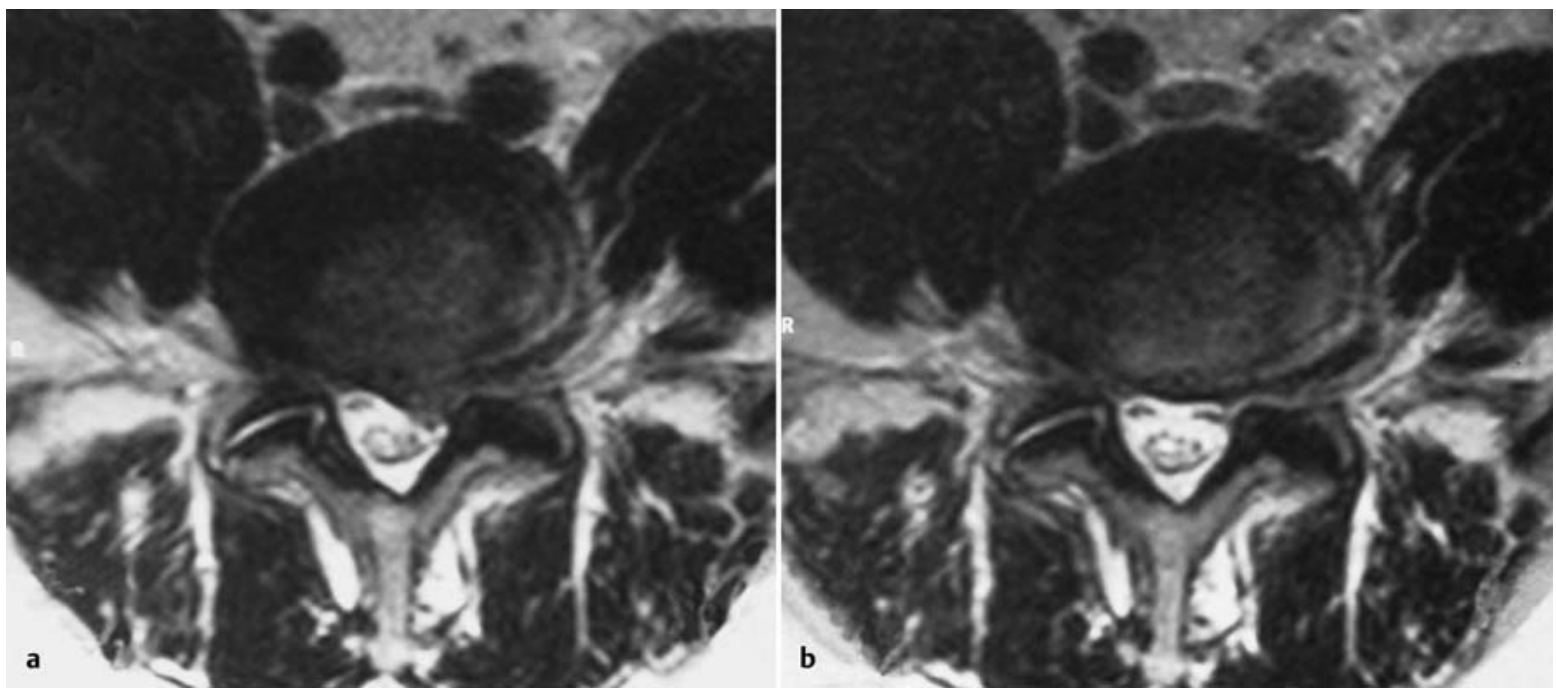

Abb. 16 - Bandscheibenprolaps L4-L5 links. a Erstdiagnostik. b Spontane Resorption nach 4 Monaten. 


\section{Konservative Therapie}

Das Therapieziel (s. o.: Infobox) gilt sowohl für die konservative als auch operative Therapie. Liegt keine Notfalloder dringliche OP-Indikation vor (s.o.), so erfolgen zunächst konservative Therapiemaßnahmen mit dem Ziel der Schmerzlinderung.

Die Rückbildung eines Bandscheibenvorfalls kann durch konservative Therapiemaßnahmen nicht sicher beeinflusst werden. Daher sind alle konservativen Therapiemaßnahmen auf die Besserung oder Rückbildung der Symptome ausgerichtet.

In der akuten Schmerzphase kann eine kurze Bettruhephase (<48 Stunden) indiziert sein, kombiniert mit oraler, i.v. oder i.m. Schmerzmedikation nach WHO-Schema (Abb. 17) bzw. bildwandler-(BV-)gestützten periradikulären oder epiduralen Injektionen. Die Gabe von Muskelrelaxanzien ist v.a. bei begleitenden Muskelverspannungen und Fehlhaltungen sinnvoll.
Leichte Traktion zur Entlastung des Bandscheibenraums als unterstützende Maßnahme kann ebenso sinnvoll sein wie lokale Wärmeapplikation (Fango, Rotlicht, Heizkissen). Stufenbettlagerung entspannt den komprimierten Spinalnerv.

Mit abklingender Beschwerdesymptomatik sollte die physiotherapeutische Behandlung über isometrische und entspannende Übungen schrittweise in ein Muskeltrainingsprogramm übergehen.

Unter diesen Maßnahmen sollte es zu einer linearen Besserung der Symptomatik kommen und nach spätestens 2-4 Wochen Beschwerdefreiheit erzielt sein.

Bei undulierendem Verlauf, fehlender linearer Besserung oder gar Verschlechterung unter konservativer Therapie sollte zeitnah ein Strategiewechsel in Erwägung gezogen werden.

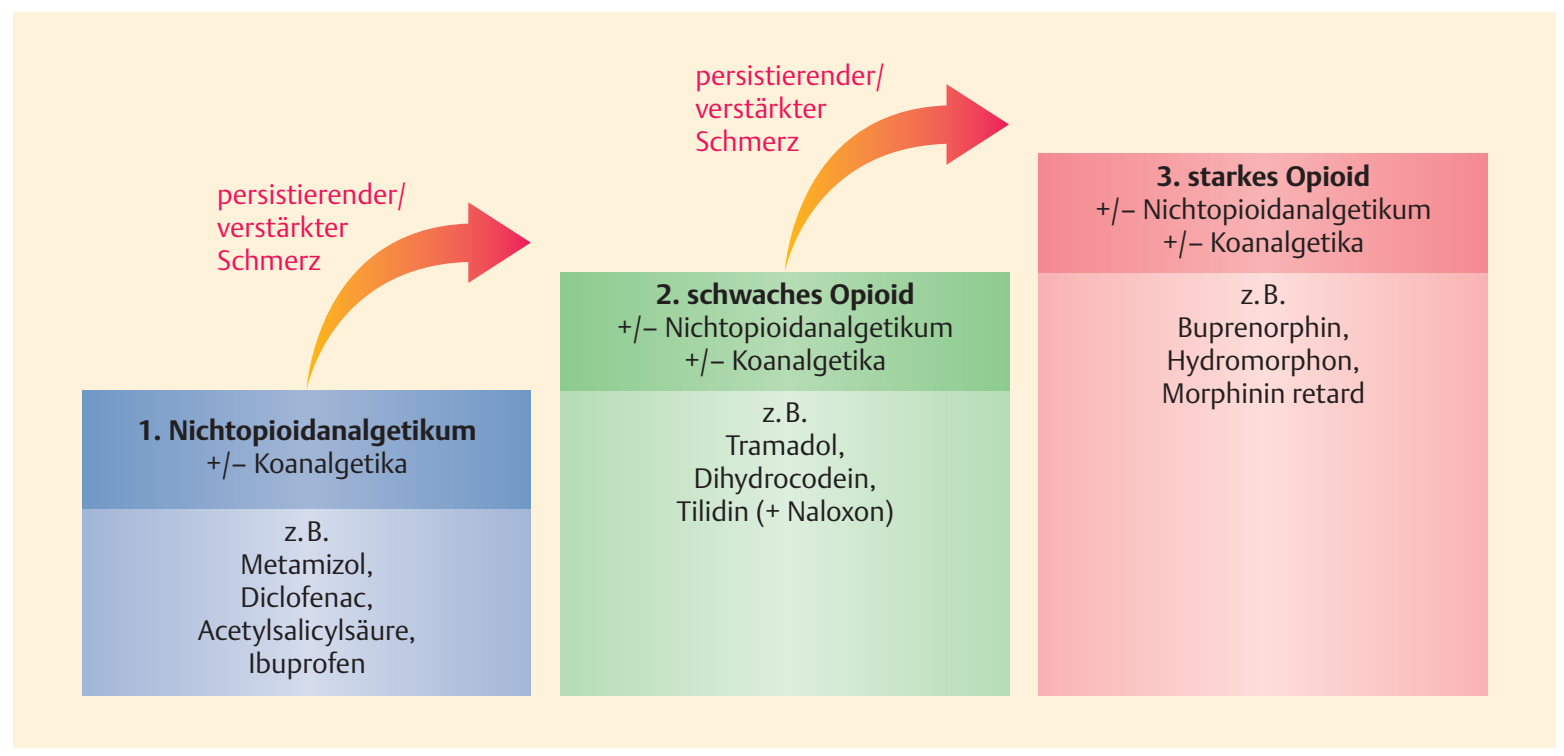

Abb. 17 - WHO-Stufenschema der Schmerztherapie. 


\section{Operation}

\section{OP-Indikation}

Bei Vorliegen einer Konus- oder Kaudasymptomatik besteht eine absolute, notfallmäßige OP-Indikation. Ähnliches gilt für rasch progrediente ( $<24$ Stunden) und schwere neurologische Ausfallerscheinungen (Kraftgrad 2/5 bis 0/5 nach Janda). Hier besteht eine dringliche OPIndikation innerhalb der nächsten 24 Stunden.

Motorische Ausfallerscheinungen Kraftgrad 3/5 und schlechter bilden sich bei einer Symptomdauer von mehr als 30 Tagen nur inkomplett zurück. Bei leichteren Paresen verlängert sich dieser Zeitraum. Kommt es innerhalb von 6-8 Wochen nicht zu einer deutlichen Verbesserung der Schmerzen sowie zu einer Zunahme der Belastungsfähigkeit, muss die Umstellung der Therapie hin zur Operation in Erwägung gezogen werden. Gleiches gilt selbstverständlich für eine sich unter konservativer Therapie verschlechternde Symptomatik. Bei ausgeprägten radikulären Schmerzen, die trotz forcierter, konservativer Therapie nicht beherrschbar sind und einen adäquaten bildmorphologischen Befund aufweisen, ist ebenfalls ein frühes operatives Vorgehen gerechtfertigt (relative OP-Indikation).

\section{Auswahl des Operationsverfahrens}

Für die Auswahl des Operationsverfahrens sowie des operativen Zugangswegs sind die Morphologie und Lokalisation des Bandscheibenvorfalls die entscheidenden Parameter.

\section{Therapieziel}

\section{Technische Operationsziele}

- sichere und definitive Dekompression des komprimierten Spinalnerven bzw. der Cauda equina

- geringes Zugangstrauma

- Erhalt der segmentalen Stabilität

- Erhalt der Bandscheibe

Die Entfernung des Bandscheibenvorfalls erfolgt in jedem Fall minimalinvasiv. Die mikrochirurgische Sequestrektomie mithilfe des OP-Mikroskops ist mittlerweile weltweit zum Standard geworden. Daneben haben sich noch weniger invasive, endoskopische Techniken etabliert, die für bestimmte Indikationen mittlerweile als Therapie der Wahl gelten.

\section{Übersicht}

\section{Minimalinvasive OP-Verfahren \\ - geschlossene, vollendoskopische Sequestrektomie (Endoskop) \\ - mikrochirurgische Operationsmethode (OP-Mikroskop)}

\section{Endoskopische Techniken}

\section{- Endoskopischer, interlaminärer Zugang}

Der interlaminäre Zugang (Abb. 18) ist für paramedian gelegene, nicht weit nach kranial oder kaudal sequestrierte Bandscheibenvorfälle geeignet, vorausgesetzt, dass ein genügend großes interlaminäres Fenster vorliegt (hauptsächlich L4-L5 und L5-S1).

\section{- Endoskopischer, transforaminaler Zugang}

Für intra- oder extraforaminale, aber auch für große medial gelegene Bandscheibenvorfälle ist der transforaminale Zugang meistens der geeignetere Weg (Abb. 19). Jedoch weist der transforaminale Zugang gegenüber dem interlaminären Zugang mehr Einschränkungen aufgrund der anatomischen Gegebenheiten auf, zeigt aber gleichzeitig die beste Gewebeschonung. Im alltäglichen Arbeiten liegt das prozentuale Verhältnis von transforaminalem im Vergleich zum interlaminären Vorgehen nach unserer Erfahrung bei ca. 35 zu 65\%.

\section{Vorsicht}

\section{Fehler, Komplikationen, Gefahren}

- Gefahr der iatrogenen Wurzel- und/oder Duraläsion

- epidurales Hämatom

- Gefahr der unzureichenden Sequesterentfernung

- mögliche Verletzungsgefahr viszeraler Organe bei zu weit ventral gelegenem Eintrittspunkt bei transforaminalen Zugängen 


\section{Vor- und Nachteile der endoskopischen Technik}

\section{Vorteile}

- kleine Hautinzision zwischen 5 und 7 mm

- atraumatischer Zugang

- minimale Instabilitätsrate, da nur in Ausnahmefällen Knochen entfernt werden muss

- permanente Lavage des OP-Gebiets antiinflammatorischer Effekt

- Redon-Drainage ist aufgrund des minimalen Zugangs nicht nötig
- kurze OP-Dauer (im Durchschnitt

\section{Minuten)}

- Same Day Surgery möglich

- geringe peri- und postoperative Morbidität

- Wundschmerz

- Mobilisation

- Rehabilitation

- gute klinische Ergebnisse

- niedrige Komplikationsraten

\section{Nachteile}

- technisch anspruchsvolles OP-Verfahren

- aufwendiges Instrumentarium

- längere Lernkurve

- eingeschränkte Möglichkeiten der OP-Erweiterung bei Komplikationen

- endoskopische Naht der Dura technisch nicht möglich

- erhöhte Strahlenbelastung (v. a. bei transforaminalen Zugängen)

- selektive Indikation
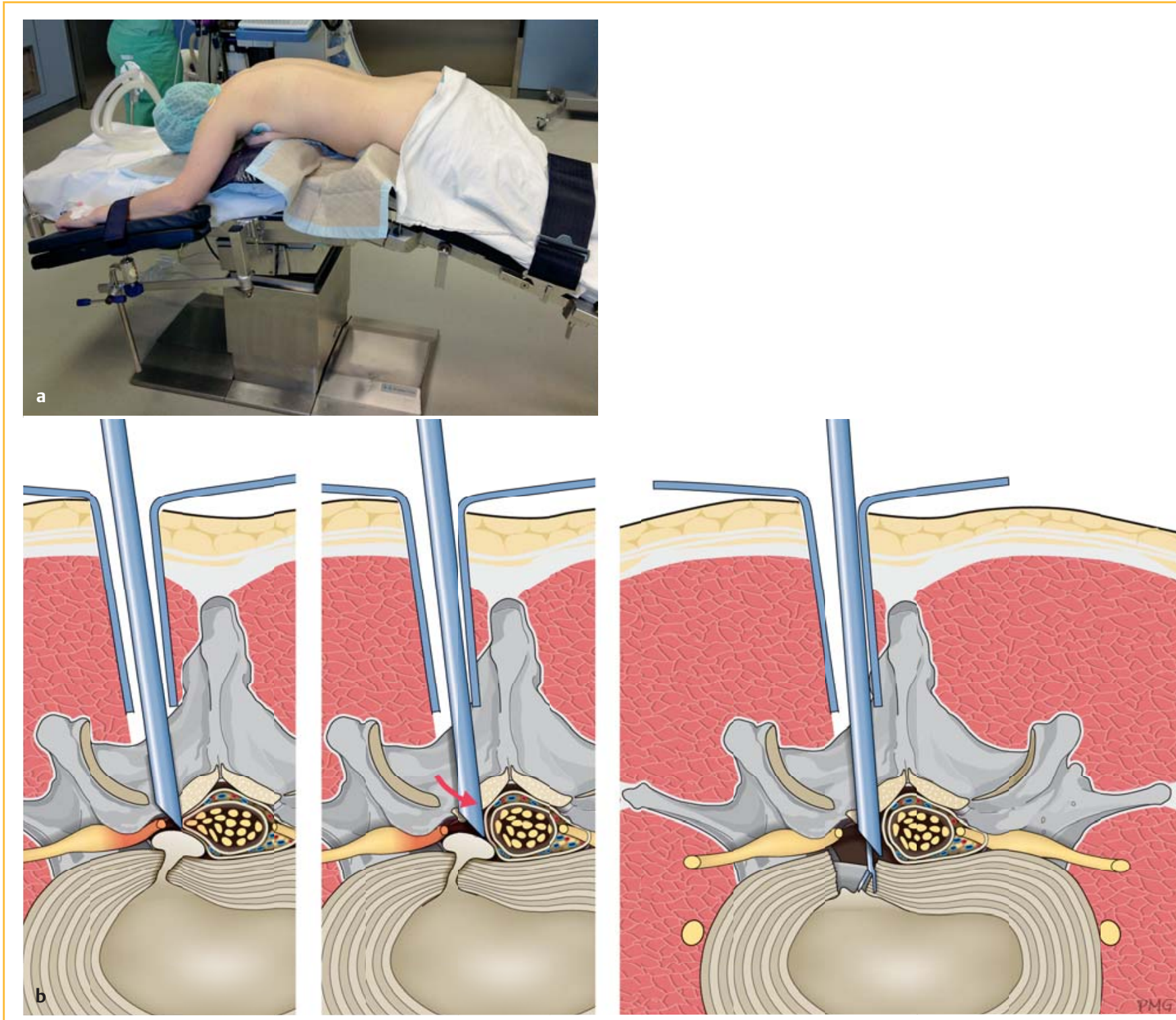

Abb. 18 - Endoskopischer interlaminärer Zugang. a Patientenlagerung. b Entfernung des Bandscheibenvorfalls mit Fasszange. 


\section{Wirbelsäule}

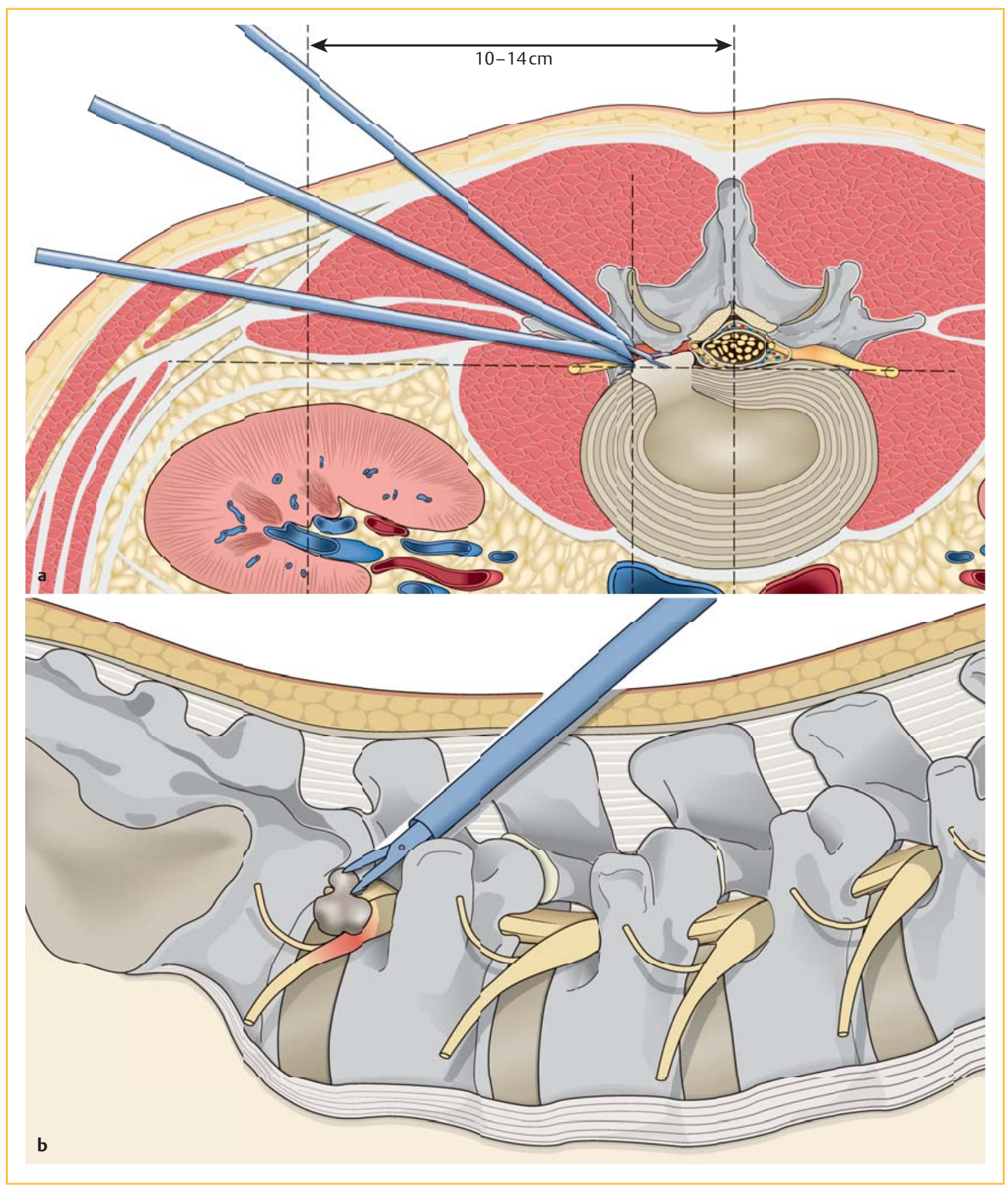

Abb. 19 - Endoskopischer Zugang transforaminal. a Platzierung der Arbeitskanüle von lateral. b Entfernung des extraforaminalen Bandscheibenmaterials. 


\section{Mikrochirurgische Techniken}

Das Operationsziel ist die Entfernung des protrudierten bzw. prolabierten/sequestrierten Bandscheibenmaterials über einen offenen (Röhrchenretraktor; Spekulumretraktor), muskelschonenden, mikrochirurgischen Zugang (OP-Mikroskop; Hautschnitt ca. 1,5-2 cm).

Es stehen, je nach Lokalisation des Bandscheibenvorfalls, 3 verschiedene Zugangswege zur Verfügung: interlaminär, extraforaminal, translaminär.

\section{- Mikrochirurgischer interlaminärer Zugang (medial und paramedian lokalisierte Bandscheibenvorfälle)}

Darstellung des interlaminären Fensters (Lig. flavum mit angrenzendem Wirbelbogen und medialem Rand des Wirbelgelenks sowie Einsetzen des Mikrospekulums bzw. Tubenretraktors; Abb. 20). Das Lig. flavum wird nun mit einem stumpfen Dissektor eröffnet. Darstellung des Spinalnervs. Dieser wird vorsichtig nach medial mobilisiert und der darunterliegende Bandscheibenvorfall dargestellt.

\section{- Mikrochirurgischer translaminärer Zugang (kranial sublaminär sequestrierte Bandscheibenvorfälle)}

Der Zugangsweg ist identisch dem interlaminären Zugang, allerdings wird der Hautschnitt leicht kranial des Bandscheibenraums platziert, sodass das Spekulum auf die Lamina positioniert werden kann. Der Hautschnitt kann auch deutlich kleiner gewählt werden (ca. 10$15 \mathrm{~mm}$ ). Intraoperative Röntgenkontrolle des Retraktors auf der Lamina. Es erfolgt nun mit der Fräse (zunächst Rosenkopf, dann Diamant) das Fräsen eines ca. $10 \mathrm{~mm}$ großen Defekts in die Lamina.

- Cave: Wichtig ist hierbei die Schonung des Isthmus interarticularis (mindestens 4-5 mm Abstand!), um postoperative Ermüdungsfrakturen zu vermeiden.

Durch den Bohrlochdefekt in der Lamina gelangt man in den Spinalkanal kranial des Zwischenwirbelraums und kann den nach kranial luxierten Bandscheibenvorfall (s. Operation/OP-Indikationen) unter Schonung des Wirbelgelenks bzw. des Lig. flavum entfernen (Abb. 21).

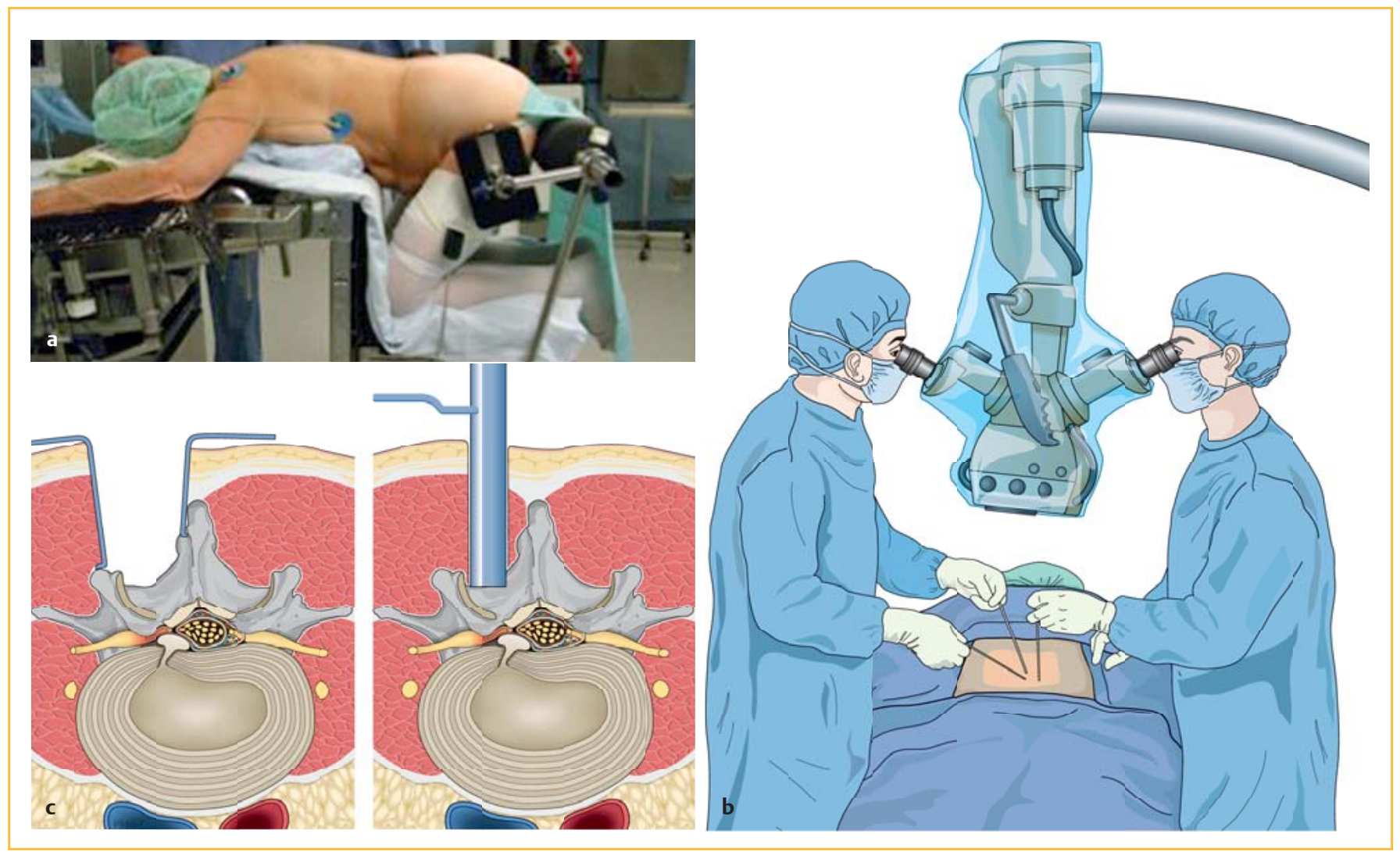

Abb. 20 - Mikrochirurgischer interlaminärer Zugang. a Patientenlagerung. b Position von Operateur und Assistent. c Paramedianer, muskelschonender Zugang mit Spekulum (links) oder Tubus (rechts). 


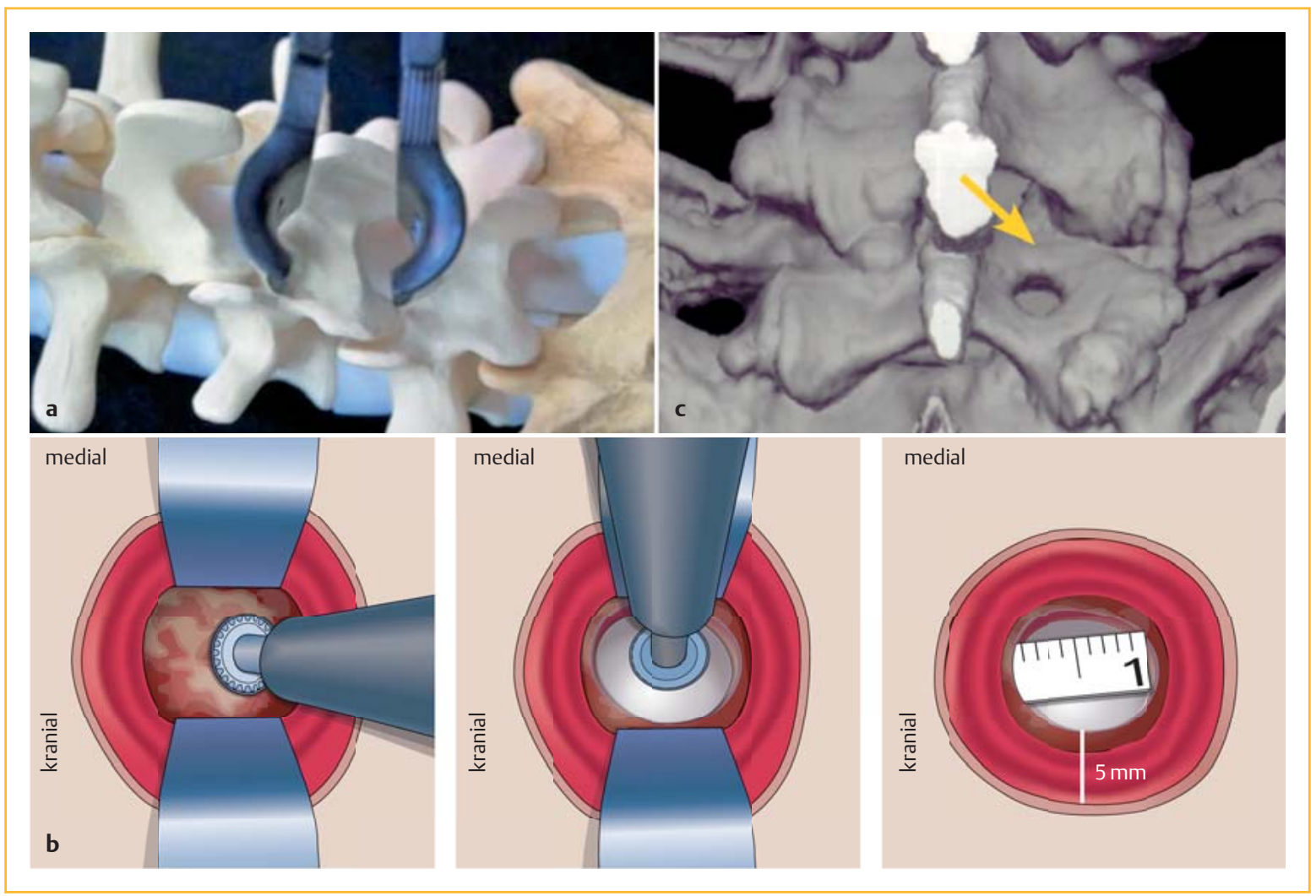

Abb. 21 - Mikrochirurgischer translaminärer Zugang. a Mikrochirurgischer translaminärer Zugang. b Translaminärer Zugang durch Fräsen einer 10-mm-Öffnung. c CT-Rekonstruktion postoperativ mit minimalem Defekt in der Lamina (Pfeil).

\section{- Mikrochirurgischer extraforaminaler Zugang (laterale intra- und extraforaminale Bandscheibenvorfälle)}

Der extraforaminale Zugang ist ein intermuskulärer (zwischen medialer und lateraler Rückenstreckergruppe) Zugang lateral des Wirbelgelenks. Der Spinalnerv (Spinalganglion) wird in seiner Austrittszone aus dem Foramen intervertebrale dargestellt (Abb. 22).

Allgemein besteht insbesondere während der Lernkurve erfahrungsgemäß wie bei allen neuen Techniken ein erhöhtes Risiko des Auftretens von Komplikationen.

\section{Vorsicht}

\section{Fehler, Komplikationen, Gefahren}

- iatrogene Wurzel- und/oder Duraläsion

- epidurale Hämatome

\section{Fazit}

Vor- und Nachteile der mikrochirurgischen Zugänge

Vorteile

- kleine Hautinzision zwischen 1,5 und $2 \mathrm{~cm}$

- atraumatischer Zugang

- minimale Instabilitätsrate, da lediglich ein schonendes und limitiertes Abtragen von Wirbelgelenk- bzw. Wirbelbogenanteil erfolgt (Diamantfräse)

- einfaches Instrumentarium

- kurze Rüstzeit

- intraoperative 3-D-Darstellung (binokulares Sehen durch OP-Mikroskop) anatomischer Strukturen

- Redon-Drainage selten nötig

- gute klinische Ergebnisse

- niedrige Komplikationsraten

- Indikation nicht durch Bandscheibenmorphologie bzw. Lokalisation limitiert (One-for-all-Verfahren)

\section{Nachteil}

- mikrochirurgische Erfahrung notwendig (Lernkurve!) 


\section{Postoperative Behandlung}

- Die postoperative Behandlung ist für beide Techniken identisch. Allerdings besitzt sie keinen signifikanten Einfluss auf das postoperative Ergebnis.

Infektionsprophylaxe. Intravenöse Single-Shot-Antibiotikagabe zur Infektprophylaxe. Bei Risikopatienten (Diabetes mellitus, Immunsuppression) erfolgt die postoperative i.v. Antibiotikatherapie über 24 Stunden.

Mobilisation. Die Erstmobilisation erfolgt i.d.R. nach Abklingen der Allgemeinanästhesie (nach ca. 4-5 Stunden). Nach Erstmobilisation lediglich schmerzbedingte Einschränkung hinsichtlich Stehen, Sitzen, Liegen und Gehen (was schmerzfrei möglich ist, ist erlaubt).

Ruhigstellung. Aufgrund langjähriger positiver Erfahrungen empfehlen wir das Tragen eines weichen Lumbalmieders (z.B. LumboTrain Bauerfeind) für 4, bei großen Anulusdefekten für 6 Wochen.

Thromboseprophylaxe. Eine Thromboseprophylaxe wird mit niedermolekularem Heparin bis zur gesicherten Vollbelastung und Vollmobilität durchgeführt.

Nahtmaterial. In der Regel erfolgt der Wundverschluss mit intrakutaner, resorbierbarer Naht. Bei anderen Nahttechniken wird das Fadenmaterial abhängig von der Wundheilung ab dem 6. postoperativen Tag entfernt.

Belastung. Nach Abschluss der Wundheilung allmählich über 6 Wochen zunehmende Belastungssteigerung. Leichtere sportliche Belastungen wie Schwimmen und Radfahren sind bereits ab der 2. postoperativen Woche erlaubt. Schweres Heben sollte in den ersten 6-8 Wochen postoperativ vermieden werden.

Arbeitsfähigkeit. Bei sitzenden Tätigkeiten ist die Arbeitsfähigkeit innerhalb von 2 Wochen postoperativ gegeben. Bei schwerer, körperlicher Arbeit sollte eine Wiedereingliederung erst ab der 7. postoperativen Woche erfolgen, um ein Frührezidiv zu vermeiden.

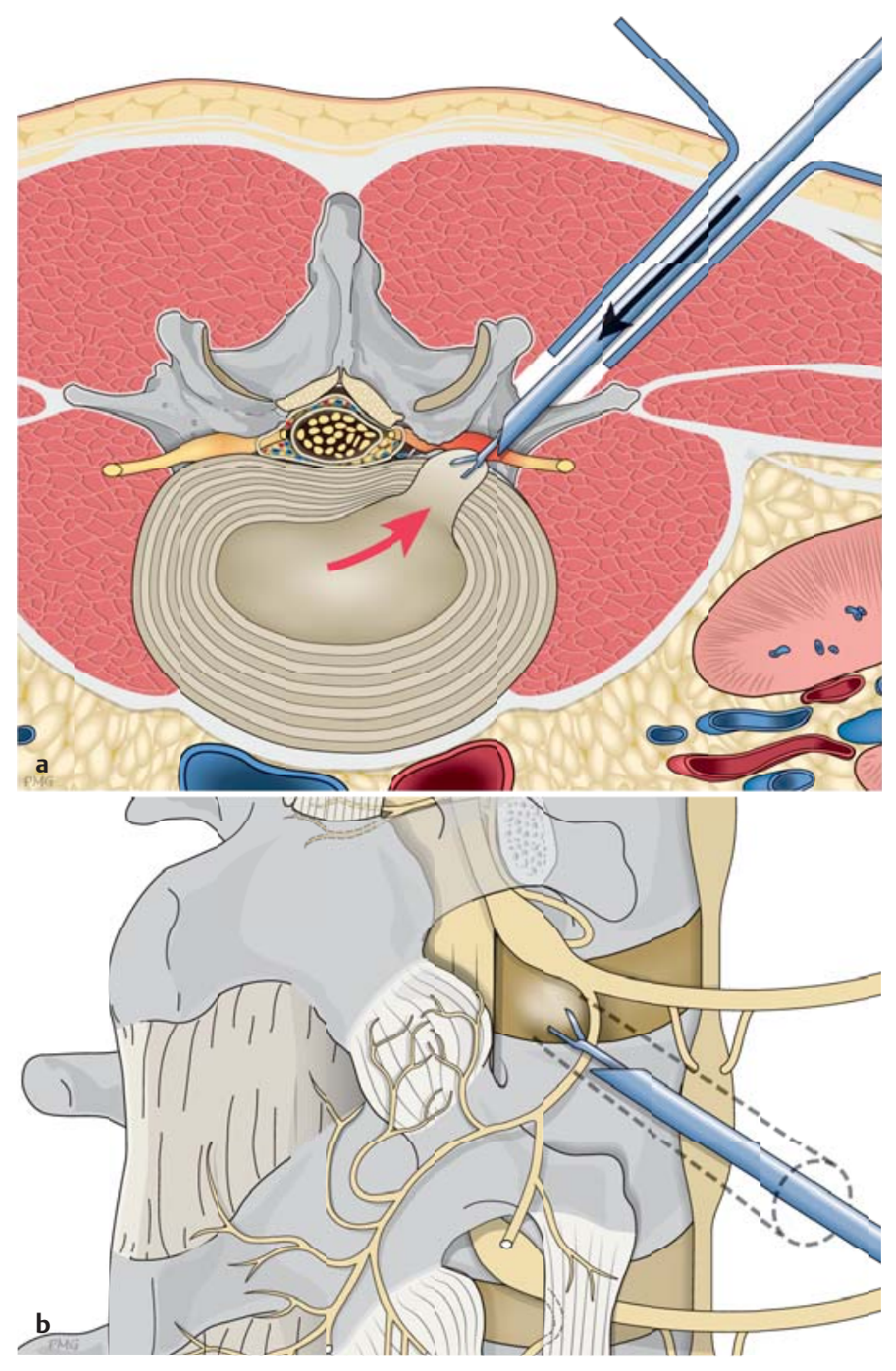

Abb. 22 - Mikrochirurgischer extraforaminaler Zugang. a Minimalinvasiver intermuskulärer Zugang. b Entfernung des Bandscheibenvorfalls extraforaminal.

\section{Prognose}

Die Wirksamkeit minimalinvasiver OP-Verfahren zur Therapie lumbaler Bandscheibenvorfälle wurde in einer Reihe prospektiver Studien inkl. Daten der Evidenzklasse 1 belegt. Die Erfolgsquoten liegen konstant für beide OP-Techniken - Endoskopie, Mikrochirurgie - im Durchschnitt zwischen 75 und 90\% bezogen auf die Rückbildung der Nervenkompressionssymptomatik. 
In einer in diesem Jahr publizierten Metaanalyse werden die klinischen Erfolgsquoten (exzellente/gute Ergebnisse) mit durchschnittlich $84,3 \%$ beziffert. $\mathrm{Zu}$ ähnlich guten Ergebnissen kommen die Publikationen der Cochrane Database 2014

Signifikante Prädiktoren für das Operationsergebnis sind allerdings

- nicht technische Faktoren wie die Symptomdauer und -schwere präoperativ sowie

- sozioökonomische und psychosoziale Faktoren.

Dennoch hat die perioperative Morbidität einen signifikanten Einfluss auf den stationären Aufenthalt, die Mobilisierung, die Rehabilitationsdauer und die Reintegration. Hier liegt der Hauptvorteil minimalinvasiver OP-Verfahren.

Bei der Rate an Rezidivbandscheibenvorfällen zeigte sich keine statistische Signifikanz zwischen beiden Gruppen (6,6\% in der vollendoskopischen Gruppe; $5,7 \%$ in der mikrochirurgischen Gruppe).

Die Langzeitprognose wird weniger durch den operativen Eingriff als vielmehr durch den allgemeinen Degenerationszustand der Lendenwirbelsäule sowie durch die genetische Prädisposition und äußere Faktoren wie Übergewicht, Rauchen und körperliche Inaktivität bestimmt.

\section{Zusammenfassung}

Lumbale Bandscheibenvorfälle zählen zu den häufigsten Krankheitsbildern an der Wirbelsäule. Genetische Prädisposition und externe Faktoren wie Übergewicht, Bewegungsmangel, Extrembelastung der Wirbelsäule sind die wichtigsten ätiologischen Faktoren. Das Häufigkeitsmaximum liegt im 4. und 5. Lebensjahrzehnt. Degenerative Veränderungen v.a. des Anulus fibrosus der Bandscheibe gehen i.d. R. einem Bandscheibenvorfall voraus. Gleiches gilt für intermittierende Rückenschmerzattacken.
Leitsymptom des lumbalen Bandscheibenvorfalls ist eine radikuläre Symptomatik durch Druck auf einen oder mehrere Spinalnerven. Die klinischen Symptome reichen von einer reinen Schmerzsymptomatik über Sensibilitätsstörungen bis hin zu motorischen Ausfallerscheinungen. In $1-1,5 \%$ der Fälle kommt es zu einer Kauda- oder Konussymptomatik mit Blasen- und oder Mastdarmstörungen oder zu rasch progredienten neurologischen Ausfallerscheinungen (<24 Stunden). Bei Paresen mit Kraftgrad 3 und schlechter kann, abhängig von den betroffenen Kennmuskeln ebenfalls eine primäre OPIndikation vorliegen. Dieses Krankheitsbild stellt eine Notfallsituation mit absoluter OP-Indikation dar. In allen anderen Fällen ist zunächst ein konservativer Therapieversuch indiziert.

Da die Rückbildung einer bestehenden Nervenkompressionssymptomatik mit der Schwere und Dauer der präoperativen Symptome korreliert, stellt sich die OPIndikation i.d.R. bei fehlender linearer Besserung der Symptomatik über einen Zeitraum von 4 Wochen bzw. bei Verschlechterung der Symptome unter konservativer Therapie. Lebensstil und Erwartungshaltung des Patienten sind weitere Faktoren, die einen Strategiewechsel von konservativer zu operativer Therapie beeinflussen können.

Die chirurgische Therapie ist minimalinvasiv (endoskopische oder mikrochirurgische Technik) mit v.a. bezüglich der Radikulopathie guten Ergebnissen.

Die Nachbehandlung ist frühfunktionell mit rascher Mobilisierung und schrittweiser Rückkehr in den beruflichen Alltag. Postoperative Rehabilitationsmaßnahmen werden häufig durchgeführt, haben jedoch keinen wissenschaftlich gesicherten Einfluss auf das Operationsergebnis.

Präventive Maßnahmen der Bandscheibendegeneration zielen auf externe Faktoren ab, wobei hier der Normalisierung des Körpergewichts im Vergleich zu allen anderen Maßnahmen eine herausragende Bedeutung zukommt.

Interessenkonflikt: In keinem Zusammenhang mit dieser Pubikation stehende wirtschaftliche Verbindungen (Beratung, Royalties) mit Depuy Synthes, Silony Medical, EIT und Paradigm Spine. 


\section{Literatur}

1 Pfirrmann CW, Metzdorf A, Zanetti M et al. Magnetic resonance classification of lumbar intervertebral disc degeneration. Spine (Phila Pa 1976) 2001; 26: 1873-1878

2 Weishaupt D, Zanetti M, Boos $\mathrm{N}$ et al. MR imaging and CT in osteoarthritis of the lumbar facet joints. Skeletal Radiol 1999; 28 :

$215-219$

\section{Zum Weiterlesen und Vertiefen}

Adams MA, McNally DS, Dolan P. Stress distributions inside intervertebral discs. The effects of age and degeneration. J Bone Joint Surg $\mathrm{Br} 1996$; 78: 965-672

Atlas SJ, Keller RB, Wu YA et al. Long-term outcomes of surgical and nonsurgical management of sciatica secondary to a lumbar disc herniation: 10 year results from the maine lumbar spine study. Spine (Phila Pa 1976) 2005; 30: 927-935

Dohrmann G], Mansour N. Long-term results of various operations for lumbar disc herniation: Analysis of over 39000 patients. Med Princ Pract 2015; 24: 285-290

Hildebrandt J, Müller G, Pfingsten M, Hrsg. Lendenwirbelsäule: Ursachen, Diagnostik und Therapie von Rückenschmerzen. München: Elsevier; 2005

Janda V. Manuelle Muskelfunktionsdiagnostik. 4. Aufl. München: Urban \& Fischer; 2000

Nakagawa H, Kamimura M, Takahara K et al. Optimal duration of conservative treatment for lumbar disc herniation depending on the type of herniation. J Clin Neurosci 2007; 14: 104-109

Pfirrmann CW, Metzdorf A, Zanetti M et al. Magnetic resonance classification of lumbar intervertebral disc degeneration. Spine (Phila Pa 1976) 2001; 26: $1873-1878$

Rasouli MR, Rahimi-Movaghar V, Shokraneh F et al. Minimally invasive discectomy versus microdiscectomy/open discectomy for symptomatic lumbar disc herniations. Cochrane Database Syst Rev 2014; 9: CD010328
Robert Koch-Institut, Hrsg. Gesundheit in Deutschland. Gesundheitsberichterstattung des Bundes. Berlin: RKI; 2006. Im Internet: http://www.gbe-bund.de/gbe10/recherche; Stand: 2013

Robert Koch-Institut, Hrsg. Rückenschmerzen. Gesundheitsberichterstattung des Bundes. Heft 53. Berlin: RKI; 2012

Weishaupt D, Zanetti M, Boos $\mathrm{N}$ et al. MR imaging and CT in osteoarthritis of the lumbar facet joints. Skeletal Radiol 1999; 28: 215-219

\section{Korrespondenzadresse}

Prof. Dr. med. H. Michael Mayer

Dr. med. Franziska Heider

Schön Klinik München-Harlaching

Akademisches Lehrkrankenhaus der Paracelsus Univ. Salzburg FIFA Medical Centre of Excellence

Wirbelsäulenzentrum

Harlachinger Str. 51

81547 München

Telefon: + $49896211-0$

E-Mail: MMayer@schoen-kliniken.de 


\section{Wirbelsäule}

\section{CME-Fragen}

\section{CME.thieme.de}

\section{CME-Teilnahme}

- Viel Erfolg bei Ihrer CME-Teilnahme unter http://cme.thieme.de

- Bitte informieren Sie sich vorab online über die Gültigkeitsdauer.

- Sollten Sie Fragen zur Online-Teilnahme haben, unter http://cme.thieme.de/hilfe finden Sie eine ausführliche Anleitung.

Eine der folgenden Behauptungen ist falsch. Welche?
1

A Lumbale Bandscheibenvorfälle zählen zu den häufigsten Wirbelsäulenpathologien.

B Ein Bandscheibenvorfall setzt häufig eine Bandscheibendegeneration voraus.

C Häufigkeitsgipfel für den Bandscheibenvorfall ist das 3.-5. Lebensjahrzehnt.

D Es besteht eine genetische Prädisposition zur Bandscheibendegeneration.

E Bandscheibenvorfälle kommen bei Kindern nicht vor.
Wodurch werden Risse im posterioren Anulus fibrosus nicht begünstigt?
A die biomechanische Belastung

B genetische Prädisposition

C Mikrotraumata

D die physiologische Kyphose der Lendenwirbelsäule

E Scherbelastungen
Wodurch wird die therapeutische Strategie bei einem Bandscheibenvorfall (BSV) bestimmt?
A die Lokalisation des BSV

B die Größe des BSV

C den betroffenen Spinalnerv

D die klinische Symptomatik und deren Verlauf

E den Rückenschmerz
Zu den erfolgversprechenden konservativen Therapiemaßnahmen bei Bandscheibenvorfall gehören ...
A kurzzeitige Bettruhe und Schmerzmedikamente.

B Analgetika und Muskelrelaxanzien.

C Wärmeapplikation und Fango.

D BV-gestützte Injektionen (periradikulär, epidural).

E alle unter A-D genannten Maßnahmen.
Die Diagnosesicherung des lumbalen Bandscheibenvorfalls erfolgt am wenigsten invasiv durch welche der im Folgenden genannten Untersuchungsmethoden?
A Röntgenbild
B Computertomografie
C Ultraschall
D Myelografie
E Kernspintomografie 
Eine der folgenden Aussagen trifft nicht zu. Welche?
A Die schnellstmögliche operative Entlastung eines Bandscheibenvorfalls sollte angestrebt werden bei einer Symptomkombination aus Harn- und Stuhlinkontinenz, Reithosenanästhesie, motorischen Ausfallerscheinungen in der unteren Extremität, radikulären Schmerzen.

B In den Epiduralraum sequestrierte Bandscheibenvorfälle zeigen eine geringere Rückbildungstendenz als Bandscheibenprotrusionen (Anulus fibrosus intakt).

C Konservative Maßnahmen erreichen bei mehr als 3/4 der Patienten mit lumbalem Bandscheibenvorfall als Erstereignis eine klinische Besserung.

D Konservative wie interventionelle und chirurgische Therapiemaßnahmen streben eine Rückbildung der radikulären Symptome und die Wiedereingliederung des Patienten in Beruf und soziales Leben an.

E Wenn unter konservativen Maßnahmen nach spätestens 1 Monat keine deutliche Symptombesserung erreicht ist, sollte ein Strategiewechsel der Therapie in Erwägung gezogen werden.
Welches sind prognostische Faktoren für ein gutes OP-Ergebnis?
A Übergewicht und Rückenschmerz
B früher OP-Zeitpunkt und leichte neurologische Ausfälle
C schwere Paresen und geringe Schmerzsymptomatik
D lange konservative Therapie (> 6 Monate) und Arbeitslosigkeit
E chronische Schmerzmedikation und Bürotätigkeit

Nur eine der folgenden Aussagen zur mikrochirurgischen Diskektomie ist korrekt. Welche?
A Die endoskopische Sequestrektomie ist bei allen Bandscheibenvorfällen möglich.

B Die endoskopische Sequestrektomie ist minimalinvasiv durchzuführen.

C Sie ist ambulant durchführbar.

D Die endoskopische Sequestrektomie führt zu vergleichbaren Ergebnissen wie die Mikrochirurgie.

E Es handelt sich um ein komplikationsarmes Verfahren.
Die postoperative Nachbehandlung bei Patienten ohne neurologische Ausfälle...
A Die mikrochirurgische Diskektomie kann nur extraforaminal durchgeführt werden.

B Es handelt sich um ein für alle Bandscheibenvorfälle adäquates Verfahren.

C Dieser Eingriff geht mit hohem Blutverlust einher.

D Die mikrochirurgische Diskektomie führt zu längeren Immobilisationszeiten.

E Es handelt sich um ein Verfahren mit hohen Rezidivraten.
A sollte bereits am OP-Tag beginnen.

B sollte mindestens 6 Wochen ambulant erfolgen.

C sollte mindestens 3 Wochen stationär erfolgen.

D hat keinen signifikanten Einfluss auf das OP-Ergebnis.

E führt zu einer deutlich schnelleren Wundheilung. 